\title{
Pinned fluxons in a Josephson junction with a finite-length inhomogeneity*
}

\author{
Gianne Derks ${ }^{\dagger}$ \\ Arjen Doelman \\ Christopher J.K. Knight\$ \\ Hadi Susanto
}

March 27, 2022

\begin{abstract}
We consider a Josephson junction system installed with a finite length inhomogeneity, either of microresistor or of microresonator type. The system can be modelled by a sine-Gordon equation with a piecewise-constant function to represent the varying Josephson tunneling critical current. The existence of pinned fluxons depends on the length of the inhomogeneity, the variation in the Josephson tunneling critical current and the applied bias current. We establish that a system may either not be able to sustain a pinned fluxon, or - for instance by varying the length of the inhomogeneity - may exhibit various different types of pinned fluxons. Our stability analysis shows that changes of stability can only occur at critical points of the length of the inhomogeneity as a function of the (Hamiltonian) energy density inside the inhomogeneity - a relation we determine explicitly. In combination with continuation arguments and Sturm-Liouville theory, we determine the stability of all constructed pinned fluxons. It follows that if a given system is able to sustain at least one pinned fluxon, there is exactly one stable pinned fluxon, i.e. the system selects one unique stable pinned configuration. Moreover, it is shown that both for microresistors and microresonators this stable pinned configuration may be non-monotonic - something which is not possible in the homogeneous case. Finally, it is shown that results in the literature on localised inhomogeneities can be recovered as limits of our results on microresonators.
\end{abstract}

Keywords: Josephson junction, inhomogeneous sine-Gordon equation, pinned fluxon, stability .

AMS subject classifications: 34D35, 35Q53, 37K50 .

\section{Introduction}

In this paper we consider a sine-Gordon-type equation describing the gauge invariant phase difference of a long Josephson junction

$$
\phi_{t t}=\phi_{x x}-D \sin (\phi)+\gamma-\alpha \phi_{t}
$$

where $x$ and $t$ are the spatial and temporal variable respectively; $\phi(x, t)$ is the Josephson phase difference of the junction; $\alpha>0$ is the damping coefficient due to normal electron flow across the junction; and $\gamma$ is the applied bias current. The parameter $D$ represents the Josephson tunneling critical current, which can vary as a function of the spatial variable.

${ }^{*}$ G. Derks acknowledges a visitor grant of the Dutch funding agency NWO and the NWO-mathematics cluster NDNS ${ }^{+}$ and the hospitality of the CWI.

${ }^{\dagger}$ Department of Mathematics, University of Surrey, Guildford, Surrey, GU2 7XH (g.derks@surrey.ac.uk)

${ }^{\ddagger}$ Mathematisch Instituut, Leiden University, P.O. Box 9512, 2300 RA Leiden, the Netherlands (doelman@math.leidenuniv.nl)

${ }^{\S}$ Department of Mathematics, University of Surrey, Guildford, Surrey, GU2 7XH (christopher.knight@surrey.ac.uk)

ॠSchool of Mathematical Sciences, University of Nottingham, University Park, Nottingham, NG7 2RD (hadi.susanto@math.nottingham.ac.uk) 
When $D$ is constant (without loss of generality, we can take $D=1$ ) and there is no imposed current and dissipation, i.e., $\gamma=\alpha=0$, the system (1) is completely integrable [1] and has a family of travelling kink solutions of the form

$$
\phi(x, t)=\phi_{0}\left(\frac{x+v t+x_{0}}{\sqrt{1-v^{2}}}\right), \quad \text { with } \quad \phi_{0}(\xi)=4 \arctan \left(e^{\xi}\right) \quad \text { for any } \quad|v|<1 .
$$

In the study of Josephson junctions, this kink represents a fluxon, i.e. a magnetic field with one flux quantum $\Phi_{0} \approx 2.07 \times 10^{-15} \mathrm{~Wb}$. If there is a small induced current and dissipation but no inhomogeneity, then there is a unique travelling fluxon whose wave speed in lowest order is given by $v=\frac{\pi}{\sqrt{16(\alpha / \gamma)^{2}+\pi^{2}}}$ and no stationary fluxons exist, see, e.g., [11].

It was first suggested and shown in [22] that if the critical current $D$ is locally perturbed, stationary fluxons can exist even if an imposed current is present $(\gamma \neq 0)$ and that a traveling fluxon (2) can be pinned by the inhomogeneity. This phenomenon is of interests from physical and fundamental point of view because such an inhomogeneity could be present in experiments due to the nonuniformity in the width of the transmission Josephson junction line (see, e.g., $[2,28]$ ) or in the thickness of the oxide barrier between the superconductors forming the junction (see, e.g., [29, 32]). About a decade after the first analysis of this phenomenon, it is shown in [17] that the interaction between a soliton and an inhomogeneity can be non-trivial, i.e. an attractive impurity, which is supposed to pin an incoming fluxon, could totally reflect the soliton provided that there is no damping in the system. Recently it is proven that the final state at which a soliton exits a collision depends in a complicated fractal way on the incoming velocity [12].

So far almost all of the analytical and theoretical work describes the local inhomogeneity by a delta-like function $[12,16,17,22]$. Yet, the length of an inhomogeneity in real experiments is varying from $0.5 \lambda_{J}[29]$ to $5 \lambda_{J}[2,28]$, with $\lambda_{J}$ being the Josephson penetration depth. Therefore, such inhomogeneities are not well described by delta-functions. Kivshar et al. [15] have considered the time-dependent dynamics of a Josephson fluxon in the presence of this more realistic setup, i.e. fluxon scatterings that take into account the finite size of the defect, within the framework of a perturbation theory, i.e., when $\alpha, \gamma$ are small and $D \approx 1$. Piette and Zakrzewski [26] recently studied the scattering of the fluxon on a finite inhomogeneity, extending $[17,12]$ for finite length defects in the case when neither applied bias current nor dissipation is present.

In this paper, we consider the problem of a long Josephson junction with a finite-length inhomogeneity and give a systematic analysis of the existence and stability of stationary fluxons, i.e., the pinned fluxons, as they lie at the heart of the interaction of the travelling fluxons with the inhomogeneity. The Josephson tunneling critical current (denoted by $D$ in (1)) is a function of space and is modelled by the step-function

$$
D(x ; L, d)= \begin{cases}d, & |x|<L, \\ 1, & |x|>L .\end{cases}
$$

with $d \geq 0$. The inhomogeneity, as modelled by (3), can be fabricated experimentally with a high controllability and precision, such that the strength and the length of the defect $d$ and $2 L$ can be made as one wishes (see $[33,34]$ and references therein for reviews of the experimental setups). When the parameter $d$ is greater or less than one, the inhomogeneity is called a microresonator respectively microresistor. They can be thought of as a locally thinned respectively thickened junction, which provide less respectively more resistance for the Josephson supercurrent to go across the junction barrier. Note that as (1) without inhomogeneity is translationally invariant, it does not matter where the inhomogeneity is placed. The existence and stability problem of pinned fluxons in finite Josephson junctions with inhomogeneity (3) has been considered numerically by Boyadjiev et al. [3, 6, 7]. Here we consider an infinitely long Josephson junction with inhomogeneity (3) and provide a full analytical 
study of the existence and stability of pinned fluxons, using dynamical systems techniques, Hamiltonian systems ideas, and Sturm-Liouville theory.

For the existence of the pinned fluxons we observe that, as $D \equiv 1$ for $|x|$ large, it follows immediately that the asymptotic fixed points of (1) are given by $\sin \phi=\gamma$, and the temporally stable stationary uniform solutions are $\phi=\arcsin \gamma+2 k \pi$. Bu definition, a pinned fluxon is a stationary solution of (1), which connects $\arcsin \gamma$ and $\arcsin \gamma+2 \pi$. Hence a pinned fluxon is a solution of the boundary value problem

$$
\begin{aligned}
& \phi_{x x}-D(x ; L, d) \sin \phi+\gamma=0 ; \\
& \lim _{x \rightarrow \infty} \phi(x)=\arcsin \gamma+2 \pi \text { and } \lim _{x \rightarrow-\infty} \phi(x)=\arcsin \gamma .
\end{aligned}
$$

First we observe that pinned fluxons can only exist for bounded values of the applied bias current, $|\gamma| \leq 1$ (where this upperbound is directly related to our choice to set $D \equiv 1$ outside the defect). Moreover, there are symmetries in this system. If $\phi(x)$ is a pinned fluxon connecting arcsin $\gamma$ (at $x \rightarrow-\infty)$ and $\arcsin \gamma+2 \pi$ (at $x \rightarrow+\infty)$, then $\phi(-x)$ is a solution as well, connecting $\arcsin \gamma+2 \pi$ $(x \rightarrow-\infty)$ and $\arcsin \gamma(x \rightarrow+\infty)$. So the second solution is a pinned anti-fluxon. The symmetry implies that we can focus on pinned fluxons and all results for pinned anti-fluxons follow by using the symmetry $x \rightarrow-x$. Another important symmetry is

$$
\phi(x) \rightarrow 2 \pi-\phi(-x) \text { and } \gamma \rightarrow-\gamma .
$$

Thus if $\phi(x)$ is a pinned fluxon with bias current $\gamma$, then $2 \pi-\phi(-x)$ is a pinned fluxon with bias current $-\gamma$. This means that we can restrict to a bias current $0 \leq \gamma \leq 1$ and the case $-1 \leq \gamma<0$ follows from the symmetry above.

Furthermore, the differential equation in (4) is a (non-autonomous) Hamiltonian ODE with Hamiltonian

$$
H=\frac{1}{2} p^{2}-D(x ; L, d)(1-\cos \phi)+\gamma \phi, \quad \text { where } \quad p=\phi_{x} .
$$

The non-autonomous term has the form of a step function, which implies that on each individual interval $(-\infty,-L),(-L, L)$, and $(L, \infty)$ the Hamiltonian is fixed, though the value of the Hamiltonian will vary from interval to interval. Therefore the solutions of (5) can be found via a phase plane analysis, consisting of combinations of the phase portraits for the system with $D=1$ and $D=d$, see also [30] for a similar approach to get existence of $\pi$-kinks. In the phase plane analysis, the length of the inhomogeneity $(2 L)$ is treated as a parameter. For $x<-L$, the pinned fluxon follows one of the two unstable manifolds of fixed point $(\arcsin \gamma, 0)$ of the reduced ODE (4). Similarly, for $x>L$ the pinned fluxon follows one of the stable manifolds of the fixed point $(\arcsin \gamma+2 \pi, 0)$. Finally, for $|x|<L$ the pinned fluxon corresponds to a part of one of the orbits of the phase portrait for the system with $D=d$. The freedom in the choice of the orbit in this system implies the existence of pinned fluxons for various lengths of the inhomogeneity. See Figure 1 for an example of the construction of a pinned fluxons when $\gamma=0.15$ and $d=0.2$. Orbits of a Hamiltonian system can be characterised by the value of the Hamiltonian, hence there is a relation between the value of the Hamiltonian inside the inhomogeneity and the length of the inhomogeneity. The resulting pinned fluxon is in $H^{2}(\mathbb{R}) \cap C^{1}(\mathbb{R})$. As the ODE (4) usually implies that the second derivative of the pinned fluxon will be discontinuous, this is also the best possible function space for the pinned fluxon solutions.

After analysing the existence of the pinned fluxons and having found a plethora of possible pinned fluxons when a bias current is applied to the Josephson junction (i.e., $\gamma \neq 0$ ), we will consider their stability. First we will consider linear stability. To derive the linearised operator about a pinned fluxon $\phi_{\text {pin }}(x ; L, \gamma, d)$, write $\phi(x, t)=\phi_{\text {pin }}(x ; L, \gamma, d)+e^{\lambda t} v(x, t ; L, \gamma, d)$ and linearise about $v=0$ to get the eigenvalue problem

$$
\mathcal{L}_{\text {pin }} v=\Lambda v, \quad \text { where } \quad \Lambda=\lambda^{2}+\alpha \lambda,
$$




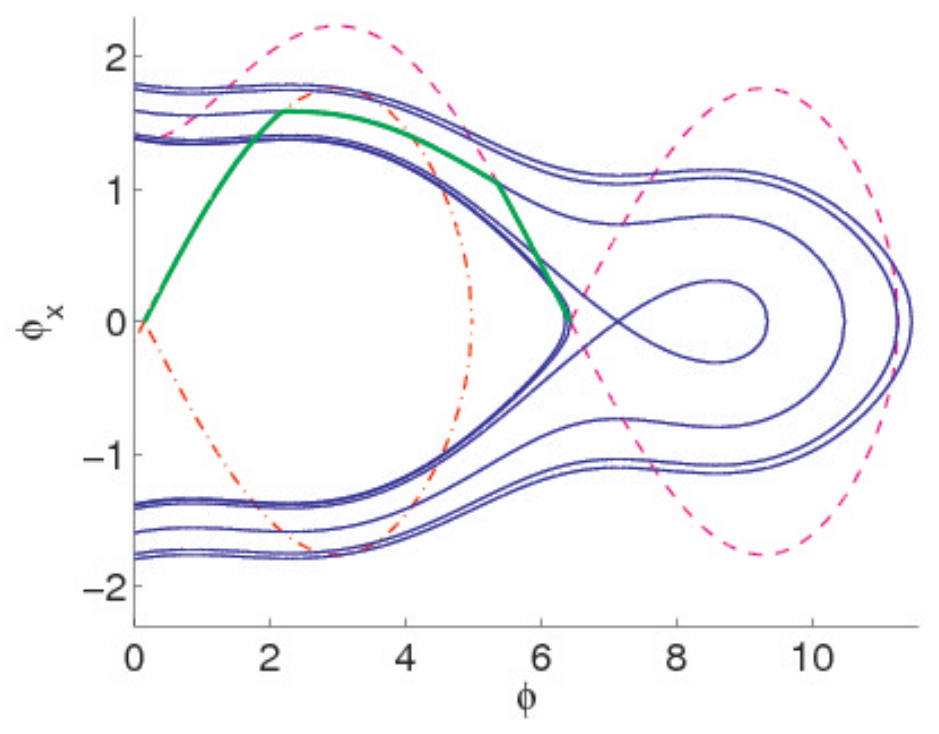

Figure 1: Phase portraits when $\gamma=0.15$ and $d=0.2$. The dash-dotted red curves are the unstable manifolds of $(\arcsin \gamma, 0)$, the dashed magenta curves are the stable manifolds of $(2 \pi+\arcsin \gamma, 0)$, and the solid blue curves are examples of orbits for the dynamcis inside the inhomogeneity. The bold green curve is an example of a pinned fluxon.

and the linearisation operator $\mathcal{L}_{\text {pin }}(x ; L, \gamma, d)$ is

$$
\mathcal{L}_{\text {pin }}(x ; L, \gamma, d)=D_{x x}-D \cos \phi_{\operatorname{pin}}(x ; L, \gamma, d)=\left\{\begin{array}{cc}
D_{x x}-\cos \phi_{\operatorname{pin}}(x ; L, \gamma, d), & |x|>L \\
D_{x x}-d \cos \phi_{\operatorname{pin}}(x ; L, \gamma, d), & |x|<L .
\end{array}\right.
$$

The natural domain for $\mathcal{L}_{\text {pin }}$ is $H^{2}(\mathbb{R})$. We call $\Lambda$ an eigenvalue of $\mathcal{L}_{\text {pin }}$ if there is a function $v \in H^{2}(\mathbb{R})$, which satisfies $\mathcal{L}_{\text {pin }}(x ; L, \gamma, d) v=\Lambda v$. This operator is self-adjoint, hence all eigenvalues will be real. Furthermore, it is a Sturm-Liouville operator, thus the Sobolev Embedding Theorem gives that the eigenfunctions are continuously differentiable functions in $H^{2}(\mathbb{R})$. Sturm's Theorem [31] can be applied, leading to the fact that the eigenvalues are simple and bounded from above. Furthermore, if $v_{1}$ is an eigenfunction of $\mathcal{L}_{\text {pin }}$ with eigenvalue $\Lambda_{1}$ and $v_{2}$ is an eigenfunction of $\mathcal{L}_{\text {pin }}$ with eigenvalue $\Lambda_{2}$ with $\Lambda_{1}>\Lambda_{2}$, then there is at least one zero of $v_{2}$ between any pair of zeros of $v_{1}$ (including the zeros at $\pm \infty$ ). Hence, the eigenfunction $v_{1}$ has a fixed sign (no zeros) if and only if $\Lambda_{1}$ is the largest eigenvalue of $\mathcal{L}_{\text {pin }}$. The continuous spectrum of $\mathcal{L}_{\text {pin }}$ is determined by the system at $\pm \infty$. A short calculation shows that the continuous spectrum is the interval $\left(-\infty,-\sqrt{1-\gamma^{2}}\right)$.

If the largest eigenvalue $\Lambda$ of $\mathcal{L}_{\text {pin }}$ is not positive or if $\mathcal{L}_{\text {pin }}$ does not have any eigenvalues, then the pinned fluxon is linearly stable, otherwise it is linearly unstable. This follows immediately from analysing the quadratic $\Lambda=\lambda^{2}+\alpha \lambda$. If $\Lambda \leq 0$, then both solutions $\lambda$ have non-positive real part. However, if $\Lambda>0$ is then there is a solution $\lambda$ with positive real part. Furthermore, the $\lambda$-values of the continuous spectrum also have non-positive real part as the continuous spectrum of $\mathcal{L}_{\text {pin }}$ is on the negative real axis.

The linear stability can be used to show nonlinear stability. The Josephson junction system without dissipation is Hamiltonian. Define $P=\phi_{t}, u=(\phi, P)$, then the equations (1) can be written as a Hamiltonian dynamical system with dissipation on an infinite dimensional vector space of $x$-dependent functions, which is equivalent to $H^{1}(\mathbb{R}) \times L^{2}(\mathbb{R})$ :

$$
\frac{d}{d t} u=\mathbf{J} \delta \mathcal{H}(u)-\alpha \mathbf{D} u, \quad \text { with } \quad \mathbf{J}=\left(\begin{array}{cc}
0 & 1 \\
-1 & 0
\end{array}\right), \quad \mathbf{D}=\left(\begin{array}{cc}
0 & 0 \\
0 & 1
\end{array}\right),
$$


and

$$
\begin{aligned}
\mathcal{H}(u)= & \frac{1}{2} \int_{-\infty}^{\infty}\left[P^{2}+\phi_{x}^{2}+2 D(x ; L, d)\left(\sqrt{1-\gamma^{2}}-\cos \phi\right)\right] d x \\
& -\gamma \int_{0}^{\infty}[\phi-\arcsin \gamma-2 \pi] d x+\gamma \int_{-\infty}^{0}[\phi-\arcsin \gamma] d x .
\end{aligned}
$$

Here we have chosen the constants terms in the $\gamma$-integrals such that they are convergent for the fluxons. Furthermore, for any solution $u(t)$ of $(1)$, we have

$$
\frac{d}{d t} \mathcal{H}(u)=-\alpha \int_{-\infty}^{\infty} P^{2} d x \leq 0
$$

As a pinned fluxon is a stationary solution, we have $D \mathcal{H}\left(\phi_{\text {pin }}, 0\right)=0$ and the Hessian of $\mathcal{H}$ about a fluxon is

$$
D^{2} \mathcal{H}\left(\phi_{\text {pin }}, 0\right)=\left(\begin{array}{cc}
-\mathcal{L}_{\text {pin }} & 0 \\
0 & I
\end{array}\right) .
$$

If $\mathcal{L}_{\text {pin }}$ has only strictly negative eigenvalues, then it follows immediately that $\left(\phi_{\text {pin }}, 0\right)$ is a minimum of the Hamiltonian and (9) gives that all solutions nearby the pinned fluxon will stay nearby the pinned fluxon, see also [10].

After this introduction, we will start the paper with an overview of simulations for the interaction of travelling fluxons and the inhomogeneity in (1) for various values of $d, L, \gamma$ and $\alpha$. This will motivate the analysis of the existence and stability of the pinned fluxons in the following sections. We start the analysis of the existence and stability of pinned fluxons by looking at a microresistor with $d=0$. The advantage of the case $d=0$ is that several explicit expressions can be derived and technical difficulties can be kept to a minimum, while it is also representative of the general case $d<1$. It will be shown that for $\gamma=0$ there is exactly one pinned fluxon for each length of the inhomogeneity. For $\gamma>0$, a plethora of solutions starts emerging. There is a minimum and maximum length outside which the inhomogeneity cannot sustain pinned fluxons. Between the minimal and the maximal length there are at least two pinned fluxons, often more. At each length between the minimum and maximum, there is exactly one stable pinned fluxon. If the length of the interval is (relatively) large, the stable pinned fluxons are non-monotonic. Note that stable non-monotonic fluxons are not possible in homogeneous systems, since for a homogeneous system the derivative of the fluxon is an eigenfunction for the eigenvalue zero of the operator associated with the linearisation about the fluxon. If the fluxon is non-monotonous, then this eigenfunction has zeros. As the linearisation operator is a Sturm-Liouville operator, this implies that the operator must have a positive eigenvalue as well, hence the non-monotonous fluxon is unstable. However, for inhomogeneous systems, the derivative of the fluxon is usually not differentiable, hence cannot give rise to an eigenvalue zero (since the eigenfunctions have to be $C^{1}$ ) and stable non-monotonic fluxons are in principle possible. This shows that the inhomogeneity can give rise to qualitatively different fluxons.

For the existence analysis of the pinned fluxons, the length of the inhomogeneity will be treated as a parameter. The pinned fluxons satisfy an inhomogeneous Hamiltonian ODE whose Hamiltonian is constant inside the inhomogeneity. It will be shown that the existence and type of pinned fluxons can be parametrised by the value of this Hamiltonian. The length of the inhomogeneity is determined by the value of the Hamiltonian and the type of pinned fluxon, leading to curves relating the length $2 L$ and the value of the Hamiltonian inside the inhomogeneity. In [19] it is shown, in the general setting of an inhomogeneous wave equation, that changes in stability of the pinned fluxons can be associated with critical points of the length function relating $L$ and the value of the Hamiltonian. The results of this paper together with Sturm-Liouville theory give the stability properties of the pinned fluxons in the general setting. 
After giving full details for the case $d=0$, for which the stability issue can be settled in dependent of [19], an overview of the results for $d>0$ is given. The general microresistor case $(0<d<1)$ is very similar to the case $d=0$. The microresonator case $(d>1)$ has some different features, but the same techniques as before can be used to analyse the existence and stability. We finish the analysis of the microresonator case by looking at the special case where microresonators approximate a localised inhomogeneity. We explicitly look at microresonators with $d=\frac{\mu}{2 L}$ and $L$ very small. For $\gamma, \alpha$ and $\mu$ small, the asymptotic results from [22] are recovered. Even in the limit of localised inhomogeneities, our work generalises [22], since our methods allows us to consider $\gamma, \alpha$ and $\mu$ larger as well.

The paper concludes with some further observations, conclusions and ideas for future research.

\section{Simulations}

To put the analysis of the existence and stability of the pinned fluxons in the next sections in a wider context, we look first at simulations of the interaction of a travelling fluxon with an inhomogeneity. Recall that in absence of dissipation and induced currents $(\alpha=0=\gamma)$, the system (1) without an inhomogeneity $(D \equiv 1)$, has a family of travelling fluxon solutions $(2)$, for each wave speed $|v|<1$, while if there is a small induced current and dissipation, but no inhomogeneity, then there is a unique travelling fluxon.

First we look at the case $\alpha=0=\gamma$ (no induced current, no dissipation) and the inhomogeneity of microresistor type with $d=0$. If the length is too short, the fluxon will not be captured, but its speed will be reduced by the passage through the inhomogeneity. If the length of the inhomogeneity is sufficiently large, the travelling fluxon will be captured. Some radiation is released in this process and the fluxon "bounces" backwards and forwards around the defect, especially if the length is "just long enough". This is consistent with the results in [26] where a detailed analysis of the interaction of a fluxon with an inhomogeneity is studied in the case that no induced current and dissipation are present. An illustration is given in Figure 2. Note that the length of the defect which captures the
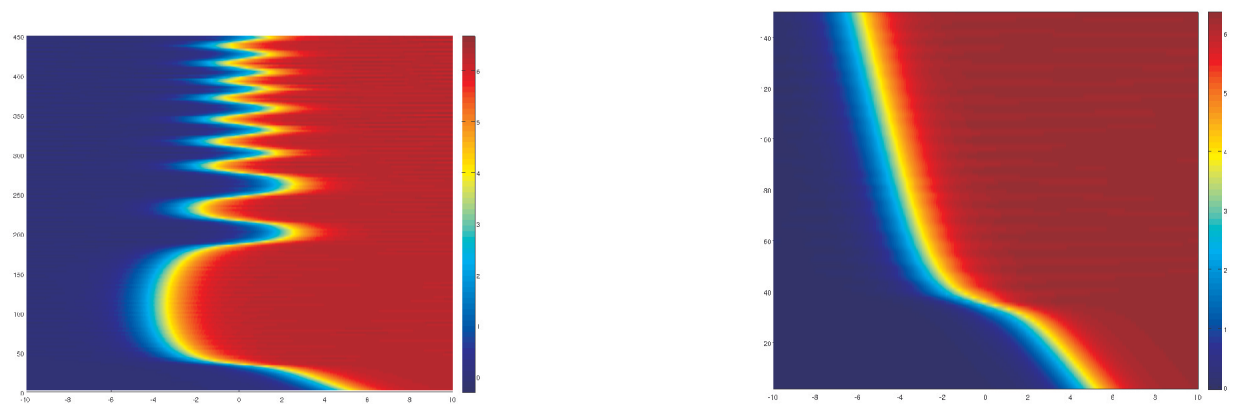

Figure 2: Simulation of a travelling wave with speed $v=0.1$ approaching an inhomogeneity with $d=0$ when there is no induced current $(\gamma=0)$ or dissipation $(\alpha=0)$. The inhomogeneity is positioned in the middle (around the zero position). The length of the inhomogeneity on the left is 0.38 and the travelling fluxon is captured by the inhomogeneity; note that the "bounce" of the fluxon is a lot larger than the length of the inhomogeneity. The length of the inhomogeneity on the right is 0.36 and the pinned fluxon can just escape, but its speed is significantly reduced.

fluxon is a lot smaller than the initial amplitude of the "bounce" of the fluxon. Observations suggest that the minimal length for the inhomogeneity to capture the travelling fluxon increases if the wave speed increases.

Next we look at the system with a microresistor with $d=0$, now with an induced current $\gamma=0.1$ and varying lengths and values of $\alpha$. We start again with an inhomogeneity of length $0.38(L=0.19)$. 
When $\gamma=0$, this microresistor captures a fluxon with speed $v=0.1$. With an induced current, it cannot capture a fluxon, however large we make $\alpha$, i.e., however slow the fluxon becomes. This is illustrated in Figure 3. The microresistor slows the fluxon down for a while, but eventually the fluxon
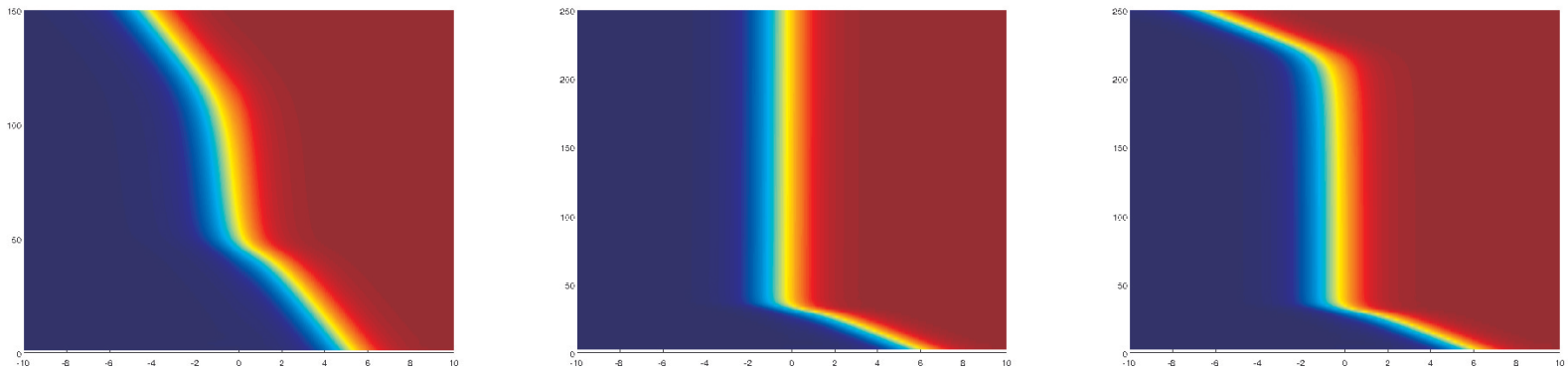

Figure 3: Simulation of a travelling fluxon approaching an inhomogeneity with $d=0$ when the induced current is $\gamma=0.1$. On the left, the length is 0.38 . Here the dissipation is $\alpha=0.9$, but however large $\alpha$ is taken, the fluxon is never captured. In the middle and right plots, the length is 0.44 . In the middle the dissipation is $\alpha=0.48$ and the fluxon is captured, on the right the dissipation is $\alpha=0.47$ and the fluxon can escape.

escapes with the same speed as it had earlier (as this speed is unique in a system with $\alpha, \gamma \neq 0$ ). The simulations suggest that the smallest length which can capture a fluxon is $0.44(L=0.22)$. In the next section, it will be shown that for $\alpha, \gamma \neq 0$, there is a minimal length under which no pinned fluxon can exist. This explains why the inhomogeneity with the shortest length cannot capture even a very slow travelling fluxon. In Figure 3 it is illustrated that, if the length can sustain pinned fluxons, the capture depends on the dissipation (hence on the speed of the incoming fluxon). If the dissipation is sufficiently large, hence the speed sufficiently slow, the pinned fluxon will be captured.

A longish defect in a microresistor will also capture the travelling wave and the resulting pinned fluxon is not monotonic, see Figure 4! The length of the inhomogeneity is substantial, so the stationary
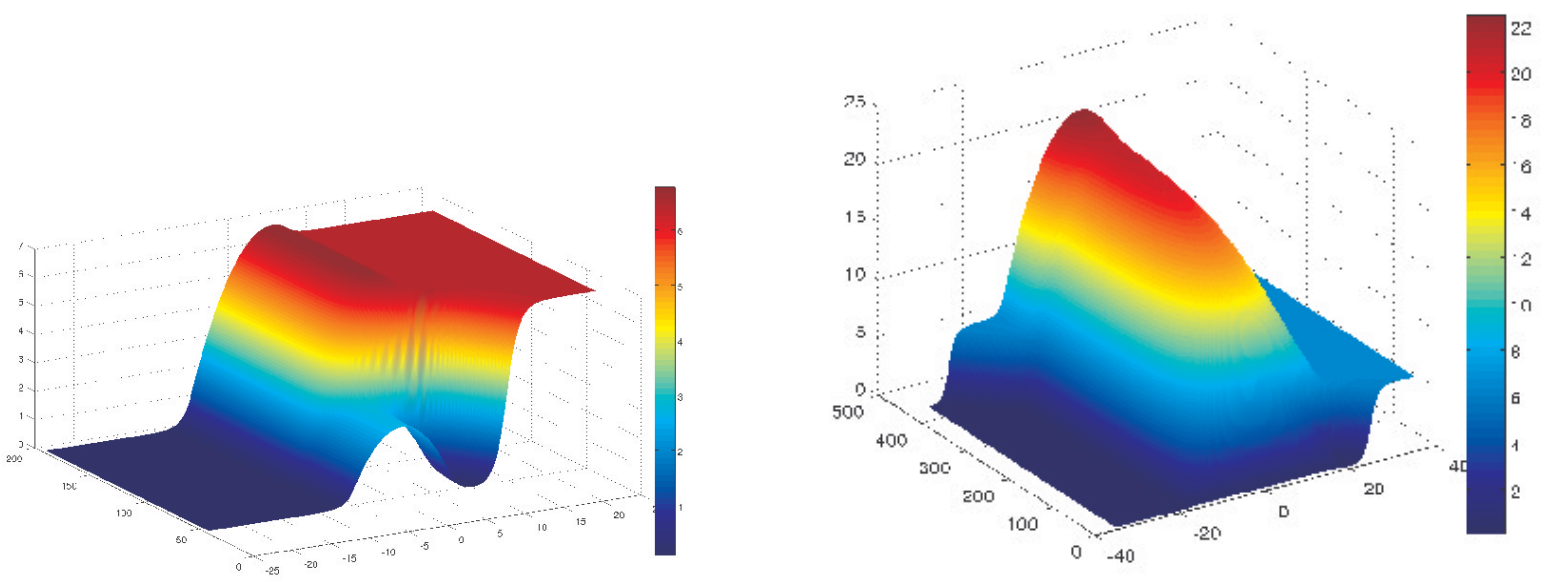

Figure 4: Simulation of a travelling fluxon approaching a longish inhomogeneity with $d=0$ when the induced current is $\gamma=0.1$ and dissipation is $\alpha=0.5$. On the left, the length is 12.5 , the travelling wave is captured and a non-monotonic pinned fluxon is formed. On the right, the length is 35 and the travelling wave escapes after a while, leaving in its wake a "bump" connecting $2 \pi+\arcsin \gamma$ at both ends. Note that the vertical scale and coloring is different in both figures; as a reference point, the travelling wave on the right is the same in both cases. 
shape connecting the far field rest states at $\arcsin \gamma$ is a "bump". This "bump" is present at all the rest states $\arcsin \gamma+2 k \pi$ for $\gamma \neq 0$ as $\arcsin \gamma+2 k \pi$ is not an equilibrium for the dynamics with $d \neq 1$. From a phase plane analysis it can be seen that the amplitude of the homoclinic connection to $\arcsin \gamma+2 k \pi$ grows with the length $L$ of the defect. As shown in Figure 4, for $L=6.25$, the travelling fluxon travels into this "bump" and gets captured. The resulting pinned fluxon is not monotonic. In the next section, the family of all possible pinned fluxons is analysed and it is shown that for long lengths the stable pinned fluxon is non-monotonic. Moreover, it follows that there is an upper limit on the length of inhomogeneities that can sustain pinned fluxons. This is illustrated on the right in Figure 4. The travelling fluxon seems to be captured initially by the inhomogeneity, but after a while it escapes again. However large the dissipation is taken, this will always happen, illustrating that no pinned fluxons can exist.

Next we consider a microresonator with $d=2$. As before, we consider the case without an induced current $(\gamma=0)$ first. In this case, the fluxon is never captured. For the smaller lengths the fluxon reflects, for larger lengths the fluxon seems to get trapped, but it escapes after a while. This is illustrated in Figure 5 for a microresonator with length 0.1. In the next section, it will be will shown that a system with a microresonator and no induced current has indeed no stable pinned fluxons.
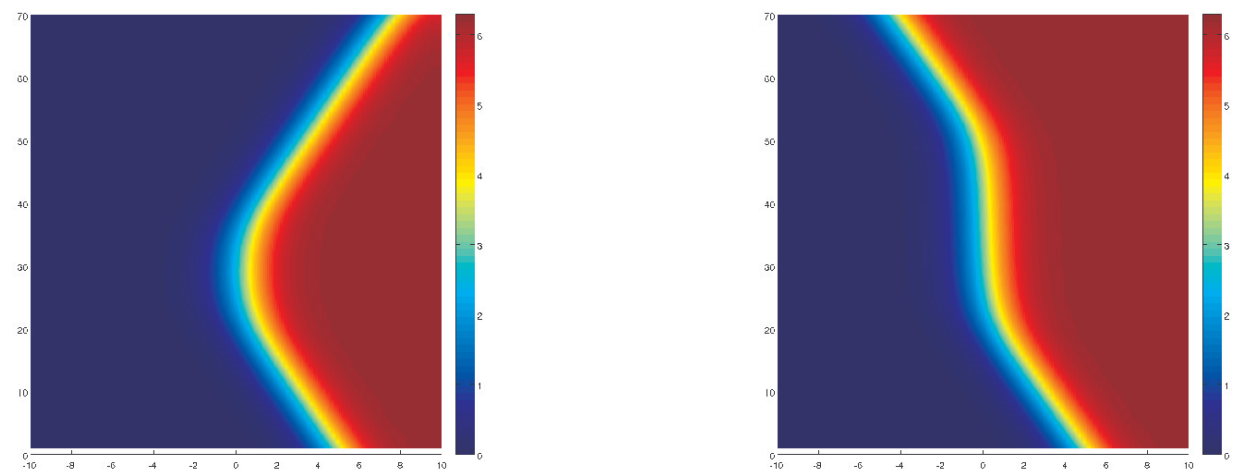

Figure 5: Simulation of a travelling wave approaching an inhomogeneity with $d=2$ and length 0.1 , when there is no induced current and no dissipation $(\gamma=0=\alpha)$. The speed on the left is 0.21 and the travelling wave is bounced by the inhomogeneity. The length on the right is 0.22 and at first the pinned fluxon seems to be captured by the inhomogeneity, but after while it travels through the inhomogeneity and seems to resume its original speed.

After the induction-less system, we consider a system with a microresonator with $d=2$ and an induced current $\gamma=0.1$. As with the microresistor there is a minimum length, under which the microresonator cannot capture a fluxon. The simulations suggest that the minimum length is 0.42 $(L=0.21)$. In Figure 6, it is illustrated that a microresonator with length 0.40 cannot capture a fluxon with $\alpha=0.9$, whilst a microresonator with length 0.42 can capture a fluxon with $\alpha=0.3$, but it cannot for $\alpha=0.29$. This is consistent with the results in the next sections where it is shown that for $\alpha, \gamma \neq 0$ there exists a minimal length under which no pinned fluxons can be sustained by the inhomogeneity. If the length can just sustain pinned fluxons, then there are both a stable and an unstable pinned fluxon close to each other. In the left panels of Figures 6 and 7 it can be observed that initially the travelling fluxon approaches the unstable pinned fluxon, but then reflects to the stable one and settles down.

Finally we consider a microresonator with a longer length for which the travelling fluxon gets captured and becomes a non-monotonic pinned fluxon. In Figure 7, it is illustrated that for a microresonator with $d=10$, length $2(L=1)$, the travelling fluxon at $\gamma=0.1$ and $\alpha=0.2$ gets attracted to a non-monotonic pinned fluxon. Note that for microresonators (i.e., $d>1$ ), the stable non-monotonic pinned fluxons have a "dip" as opposed to the ones for the microresistors which have a "bump". 

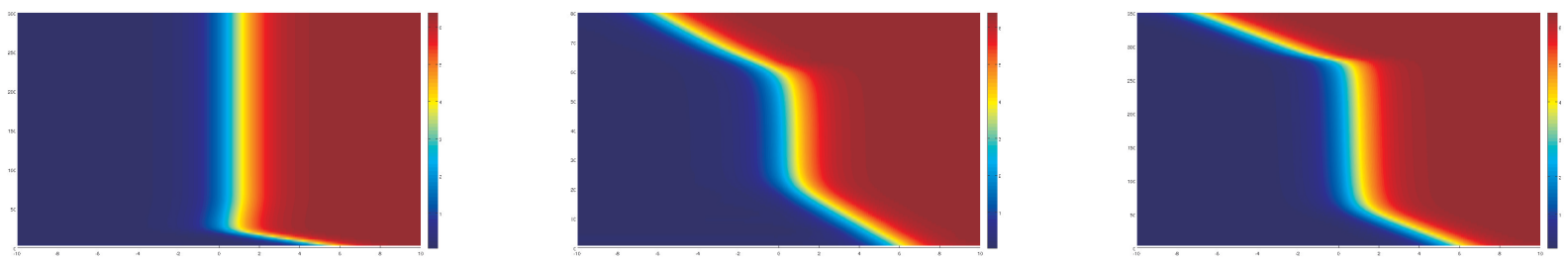

Figure 6: Simulation of a travelling wave approaching an inhomogeneity with $d=2$ and length 0.1, when there an induced current $(\gamma=0.1)$. On the left and middle is a microresonator with length 0.42 . On the left the dissipation is $\alpha=0.3$ and the fluxon is captured, whilst in the middle the dissipation is $\alpha=0.29$ and the fluxon escapes. On the right, the length is 0.4 and the dissipation is $\alpha=0.9$ and the fluxon still escapes as the length is too short for a pinned fluxon to exist.
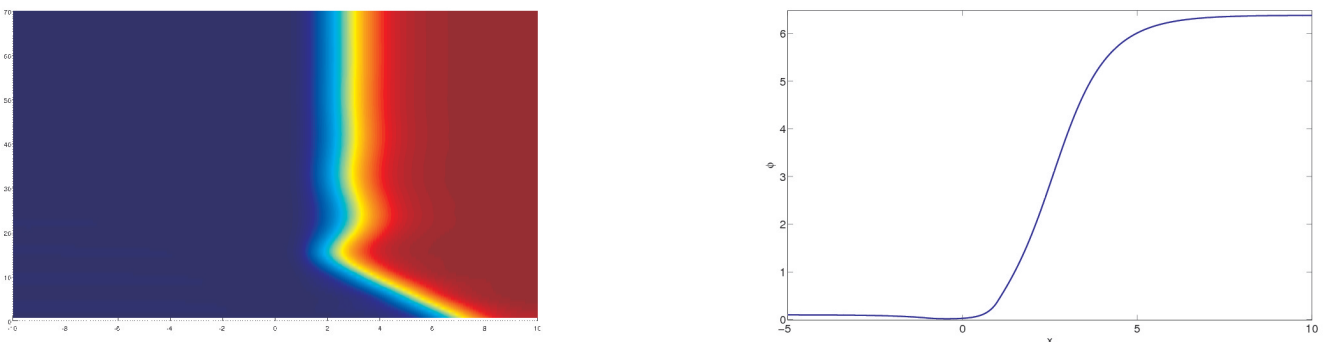

Figure 7: Simulation of a travelling wave approaching an inhomogeneity with $d=10$ and length 2, when the induced current is $\gamma=0.1$ and the dissipation is $\alpha=0.2$. The resulting wave is non-monotonic as can be seen on the right. Due to the weaker dissipation, it takes some time for the wave to converge to its stable shape. Initially, the travelling wave approaches the monotonic unstable pinned fluxon, then deflects from it and converges to the non-monotic stable one.

\section{No resistance $(\mathrm{d}=0)$}

We now analyse the existence and stability of the pinned fluxons in a microresistor and a microresonator. First we consider the case when there is no resistance in the inhomogeneity, hence a microresistor with $d=0$. This case provides a good illustration of the richness of the family of pinned fluxons, shows the essence of the analytic techniques for the existence and stability analysis, and has less technical complications than the more general values of $d$. The existence analysis for the case with no bias current $(\gamma=0)$ is quite different from the case when a bias current is applied $(\gamma>0)$. So we will consider them separately.

\subsection{Existence of pinned fluxons without applied bias current}

For $\gamma=0$, the pinned fluxon has to connect the stationary states at $\phi=0$ and $\phi=2 \pi$. In the background dynamics of the ODE (4) with $D \equiv 1$, the unstable manifold of $(0,0)$ coincides with the stable manifold of $(2 \pi, 0)$, as follows immediately by analysing the Hamiltonian $(5)$ with $D \equiv 1$. These coinciding manifolds are denoted by a red curve in the phase portrait sketched in Figure 8 . This curve represents the unperturbed sine-Gordon fluxon (2). The orbits generated by the Hamiltonian system with $D \equiv 0$ are straight lines. In Figure 8, samples of these orbits are given by the blue lines. Any blue line that crosses the red line can be used to form a pinned fluxon. An example is given in the panel on the right in Figure 8, where the green curve represents a pinned fluxon in $H^{2}(\mathbb{R}) \cap C^{1}(\mathbb{R})$.

As can be seen from Figure 8, the value of the Hamiltonian inside the inhomogeneity is a convenient parameter to characterise the pinned fluxons. The points of intersection for the blue and red curves 

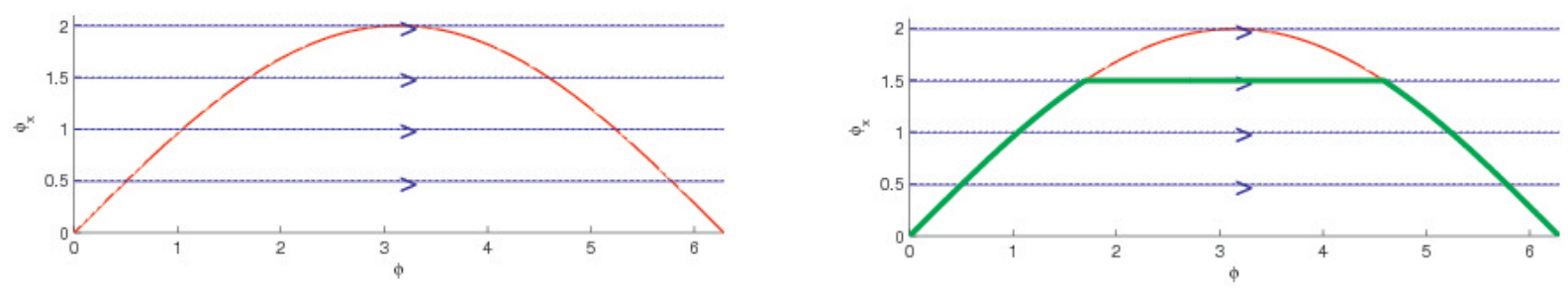

Figure 8: Phase portraits of the ODE (4) for $\gamma=0$ and $d=0$. The red curve represents the coinciding stable and unstable manifolds of the asymptotic fixed points. The blue curves are orbits for the system inside the inhomogeneity. In the sketch on the right, the green curve represents a pinned fluxon.

are denoted by $\left(\phi_{\text {in }}, p_{\text {in }}\right)$ respectively $\left(\phi_{\text {out }}, p_{\text {out }}\right)$ for the first respectively second intersection. It follows immediately that $p_{\text {in }}=p_{\text {out }}$ and $\phi_{\text {out }}=2 \pi-\phi_{\text {in }}$. Furthermore, the expression for the Hamiltonian, (5), gives the following relations for $\phi_{\text {in }}$ and $p_{\text {in }}: 0=\frac{1}{2} p_{\text {in }}^{2}-\left(1-\cos \phi_{\text {in }}\right)(D \equiv 1)$ and $h=\frac{1}{2} p_{\text {in }}^{2}(D \equiv 0)$, with $0<h \leq 2$ where $h$ is the value of the Hamiltonian inside the inhomogeneity. Thus

$$
p_{\text {in }}(h)=\sqrt{2 h} \quad \text { and } \quad \phi_{\text {in }}(h)=\arccos (1-h), \quad \text { with } \quad 0<h \leq 2 .
$$

Inside the inhomogeneity $(|x|<L)$, the pinned fluxon related to the value $h$ satisfies $h=\frac{1}{2} \phi_{x}^{2}$, thus $\phi_{x}=\sqrt{2 h}$. Hence the half-length $L$ and the parameter $h$ are related by

$$
L=\int_{-L}^{0} d x=\int_{\phi_{\mathrm{in}}(h)}^{\pi} \frac{d \phi}{\phi_{x}}=\int_{\phi_{\mathrm{in}}(h)}^{\pi} \frac{d \phi}{\sqrt{2 h}}=\frac{\pi-\arccos (1-h)}{\sqrt{2 h}} .
$$

As the numerator is a monotonic decreasing function of $h$ and the denominator is monotonic increasing, it follows immediately that $L$ is a monotonic decreasing function of $h$. The function $L$ takes values in $[0, \infty)$ as $\lim _{h \rightarrow 0} L(h)=\infty$ and $\lim _{h \rightarrow 2} L(h)=0$. The $h-L$ plot is given in Figure 9. We summarise the existence results for pinned fluxons without a bias current in the following lemma.

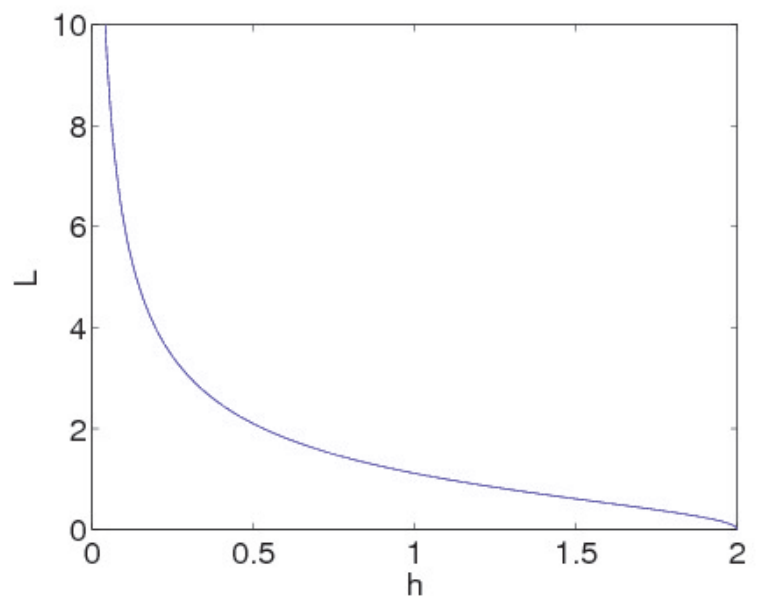

Figure 9: Plot of the length $L$ as a function of $h$, the value of the Hamiltonian in the inhomogeneity, for $\gamma=0$ and $d=0$.

Lemma 1 Let $\gamma=0$ and $d=0$. For any length $2 L$ of the inhomogeneity, there is a unique pinned fluxon, for which the Hamiltonian inside the inhomogeneity has the value $h(L)$ as implicitly given by (11). Define $x^{*}$ to be the shift such that $\phi_{0}\left(-L+x^{*}\right)=\phi_{\text {in }}$ (see (2) for the definition of $\left.\phi_{0}\right)$, then 
the pinned fluxon is given explicitly by

$$
\phi_{\text {pin }}(x ; L, 0,0)= \begin{cases}\phi_{0}\left(x+x^{*}\right), & x<-L, \\ \pi+\frac{\pi-\arccos (1-h)}{L} x, & |x|<L, \\ \phi_{0}\left(x-x^{*}\right), & x>L .\end{cases}
$$

\subsection{Existence of pinned fluxons with bias current}

For $\gamma>0$, the pinned fluxon has to connect the stationary states at $\phi=\arcsin \gamma$ and $\phi=2 \pi+\arcsin \gamma$. In the background dynamics with $D \equiv 1$ the unstable manifold of $\phi=\arcsin \gamma$ coincides no longer with the stable manifold of $2 \pi+\arcsin \gamma$. Furthermore, the orbits of the dynamics inside the inhomogeneity are parabolic curves instead of straight lines. These two changes add substantial richness to the family of pinned fluxons.

Let us first consider the phase portraits. In Figure 10 we consider $\gamma=0.15$ as a typical example to illustrate the ideas. In the dynamics with $D \equiv 1$, the unstable manifolds to $\arcsin \gamma$ are denoted by red curves, while the stable manifolds to $2 \pi+\arcsin \gamma$ are denoted by magenta curves. The larger $\gamma$ gets, the wider the gap between the unstable and stable manifold becomes. The dynamics within
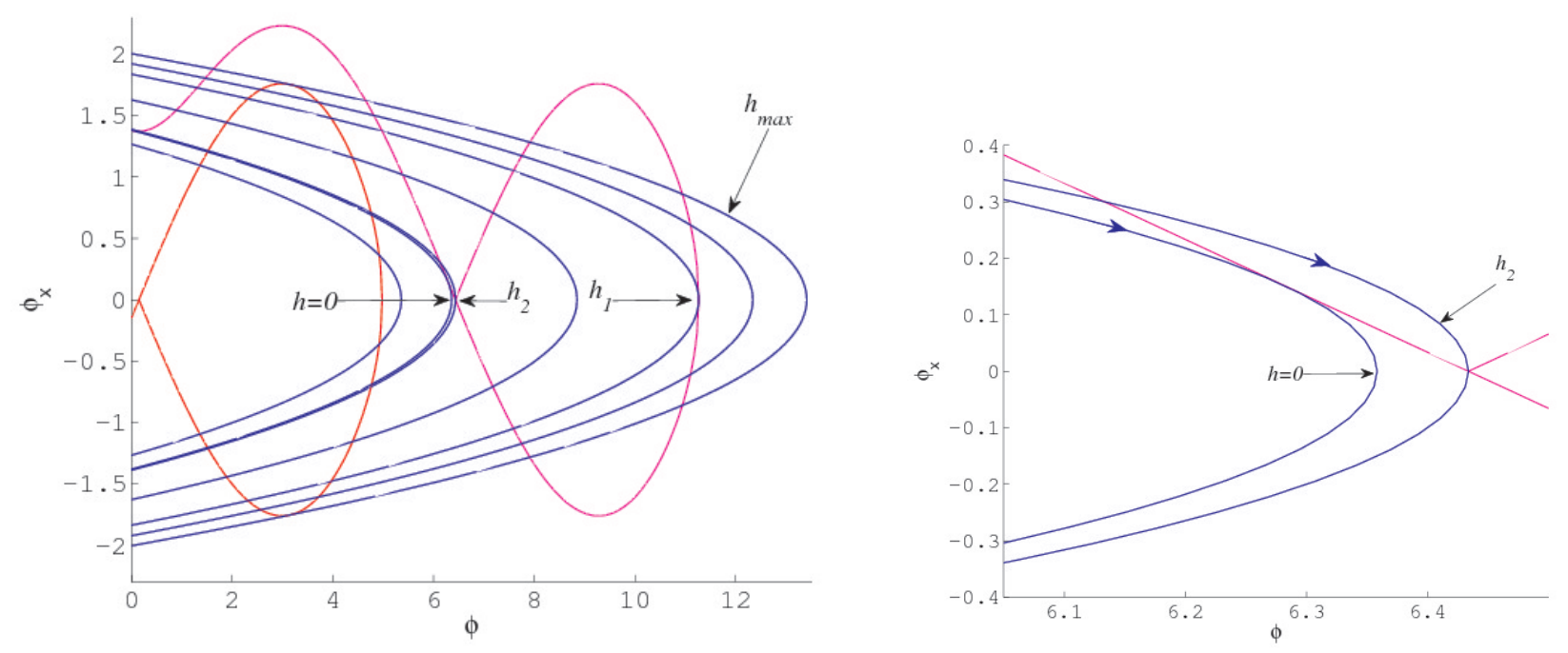

Figure 10: Phase portrait at $\gamma=0.15$ and $d=0$. On the right is a zoom into the area around $\left(\phi, \phi_{x}\right)=(2 \pi, 0)$.

the inhomogeneity with $D \equiv 0$ are denoted by blue curves. These blue curves are nested and can be parametrised with a parameter $h$, using the Hamiltonian (5) with $D \equiv 0$ :

$$
\frac{1}{2}\left(\phi_{x}\right)^{2}+\gamma \phi=H_{0}(\gamma)+h
$$

where $H_{0}(\gamma)$ is given by the value of the Hamiltonian (5) on the magenta stable manifold $(D \equiv 1)$ :

$$
H_{0}(\gamma)=\gamma \arcsin \gamma-\left(1-\sqrt{1-\gamma^{2}}\right)+2 \pi \gamma .
$$

Thus the value of $h$ increases as the extremum of the blue curves is more to the right.

For the existence of pinned fluxons, a blue curve has to connect the red unstable manifold with the magenta stable manifold. In Figure 10, the furthest left possible blue curve for which pinned fluxons may exist, is the one indicated with $h=0$. In the zoom on the right, it can be seen that this curve just touches the magenta stable manifold. The blue curve intersects the red unstable manifold twice, both points give rise to a pinned fluxon, as sketched in Figure 11. Obviously, the pinned fluxon in the 

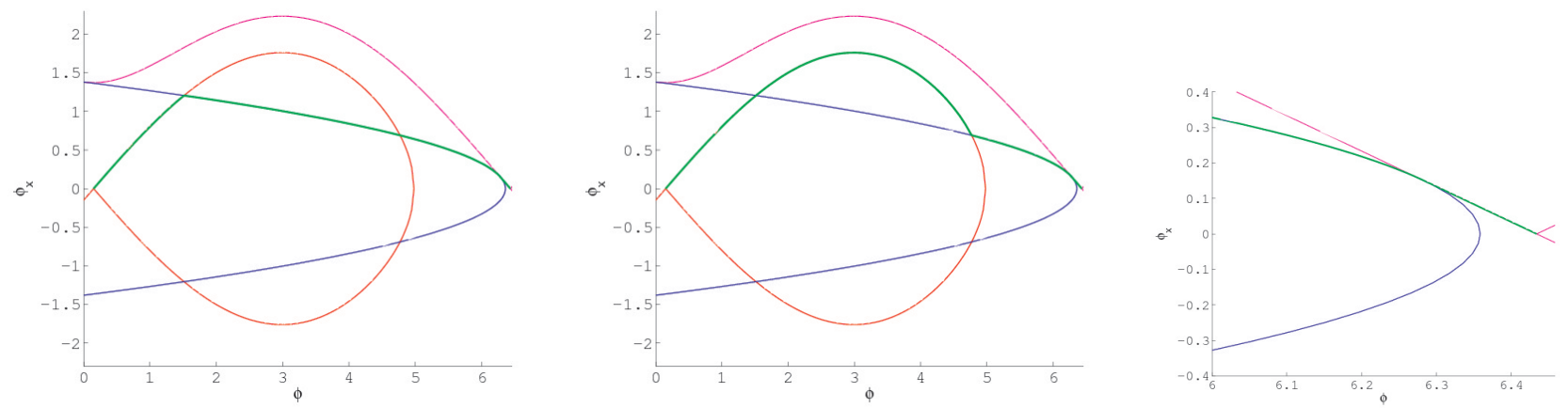

Figure 11: Phase portrait at $\gamma=0.15$ and $d=0$ with the furthest left blue curve for which pinned fluxons exist. There are two pinned fluxons possible, represented by green line. On the right is a zoom into the area around $\left(\phi, \phi_{x}\right)=(2 \pi, 0)$.

second plot in Figure 11 will occur in a defect with a shorter length than the one in the first plot. The furthest right possible curve that gives rise to pinned fluxons is marked with $h_{\max }$ in Figure 10. This blue orbit touches the red unstable manifold and crosses the magenta unstable manifolds in 5 points. All these points represent different pinned fluxons, hence 5 pinned fluxons can be associated with this curve. Moreover, for $h$ just below $h_{\max }$, the blue curve intersects the red curve twice (while it still intersects the magenta curve 5 times: there are 10 different pinned fluxons associate to such value of $h$.

In general, the pinned fluxons are determined by two points in the phase plane: the point where pinned fluxon enters the inhomogeneity (i.e. the crossing from the red unstable manifold to the blue orbit), this point will be denoted by $\left(\phi_{\text {in }}, p_{\text {in }}\right)$ and the point where the pinned fluxon leaves the inhomogeneity (i.e. the crossing from the blue orbit to the magenta stable manifold), this point will be denoted by $\left(\phi_{\text {out }}, p_{\text {out }}\right)$. Thus the points $\left(\phi_{\text {in }}, p_{\text {in }}\right)$ and $\left(\phi_{\text {out }}, p_{\text {out }}\right)$ are determined by the set of equations

$$
\begin{aligned}
H_{0}(\gamma)-2 \pi \gamma & =\frac{1}{2} p_{\text {in }}^{2}-\left(1-\cos \phi_{\text {in }}\right)+\gamma \phi_{\text {in }}, \\
H_{0}(\gamma)+h & =\frac{1}{2} p_{\text {in }}^{2}+\gamma \phi_{\text {in }}, \\
H_{0}(\gamma)+h & =\frac{1}{2} p_{\text {out }}^{2}+\gamma \phi_{\text {out }}, \\
H_{0}(\gamma) & =\frac{1}{2} p_{\text {out }}^{2}-\left(1-\cos \phi_{\text {out }}\right)+\gamma \phi_{\text {out }} .
\end{aligned}
$$

Combining the equations in (14), we get expressions for $\phi_{\text {in }}$ and $\phi_{\text {out }}$ :

$$
\cos \phi_{\text {in }}=1-(h+2 \pi \gamma) \quad \text { and } \quad \cos \phi_{\text {out }}=1-h .
$$

This is well-defined only if $0 \leq h \leq 2(1-\pi \gamma)$. Hence there are maximal values for $\gamma$ and $h$, given by

$$
\gamma_{\max }=\frac{1}{\pi} \quad \text { and } \quad h_{\max }=2(1-\pi \gamma) .
$$

If $\gamma>\gamma_{\max }$, then there is no blue curve that intersects both the red unstable orbit and the magenta stable orbit, hence no pinned fluxons exist if the applied bias current is larger than $\gamma_{\max }$. If $h>h_{\max }$, then the blue curve does not intersect the red manifold anymore.

Furthermore, $\phi_{\text {in }}$ must lie on the red unstable manifold, hence $\arcsin \gamma \leq \phi_{\text {in }} \leq \phi_{\max }(\gamma)$, where $\phi_{\max }(\gamma)$ is the maximal $\phi$-value of the orbit homoclinic to $\arcsin \gamma$. As $h \in[0,2(1-\pi \gamma)]$, this implies that there are two possible values for $\phi_{\text {in }}$ and that $p_{\text {in }}>0$ :

$$
\phi_{\text {in }}=\pi \pm \arccos (2 \pi \gamma-(1-h)) \quad \text { and } \quad p_{\text {in }}=\sqrt{2\left(H_{0}(\gamma)+h-\gamma \phi_{\text {in }}\right)} .
$$

Note that the unstable manifold left of $\arcsin \gamma$ only intersects with blue curves that have $\phi_{x}<0$, hence those orbits can never connect to one of the stable manifolds of $2 \pi+\arcsin \gamma$. 
The point $\left(\phi_{\text {out }}, p_{\text {out }}\right)$ has to lie on the magenta stable manifolds, so there can be up to five possible branches of solutions:

1. $\phi_{\text {out }}=2 \pi-\arccos (1-h)$ with $p_{\text {out }}>0$, for all $0 \leq h \leq h_{\max }$;

2. $\phi_{\text {out }}=2 \pi+\arccos (1-h)$ with $p_{\text {out }} \geq 0$, for $0 \leq h \leq h_{2}$ and $p_{\text {out }}<0$, for $h_{2}<h \leq h_{\max }$;

3. $\phi_{\text {out }}=2 \pi+\arccos (1-h)$ with $p_{\text {out }} \geq 0$, for $h_{2}<h \leq h_{\max }$;

4. $\phi_{\text {out }}=4 \pi-\arccos (1-h)$ with $p_{\text {out }} \geq 0$, for $h_{1}<h \leq h_{\max }$;

5. $\phi_{\text {out }}=4 \pi-\arccos (1-h)$ with $p_{\text {out }}<0$, for $h_{1}<h \leq h_{\max }$.

Here $h_{2}$ is the $h$-value such that the blue orbit intersects the magenta manifolds at the equilibrium $(2 \pi+\arcsin \gamma, 0)$, i.e., $h_{2}(\gamma)=1-\sqrt{1-\gamma^{2}}$, and $h_{1}$ is such that the blue orbit touches the magenta manifold at $\left(2 \pi+\phi_{\max }(\gamma), 0\right)$, the most-right point, thus $h_{1}(\gamma)=1-\cos \left(\phi_{\max }(\gamma)\right)$. In all cases, $\left|p_{\text {out }}\right|=\sqrt{2\left(H_{0}(\gamma)+h-\gamma \phi_{\text {out }}\right)}$.

To satisfy $h_{2}(\gamma) \leq h_{\max }(\gamma)$, we need that $\gamma \leq \gamma_{2}=\frac{4 \pi}{4 \pi^{2}+1} \approx 0.3104$. If $\gamma>\gamma_{2}$, then only pinned fluxons with $\phi_{\text {out }}=2 \pi \pm \arcsin \gamma$ and $p_{\text {out }}>0$ exist. In order to have $h_{1}(\gamma) \leq h_{\max }(\gamma)$, we need that $\gamma \leq \gamma_{1}$, where $\gamma_{1}$ is the implicit solution of $\cos \phi_{\max }\left(\gamma_{1}\right)+1=2 \pi \gamma_{1}$, i.e., $\gamma_{1} \approx 0.1811$. If $\gamma>\gamma_{1}$, then no pinned fluxons with $\phi_{\text {out }}=4 \pi-\arcsin \gamma$ exist. On the intervals of common existence, we have $0 \leq h_{2}(\gamma) \leq h_{1}(\gamma) \leq h_{\max }(\gamma), h_{1}\left(\gamma_{1}\right)=h_{\max }\left(\gamma_{1}\right), h_{2}\left(\gamma_{2}\right)=h_{\max }\left(\gamma_{2}\right)$, see Figure 12 .

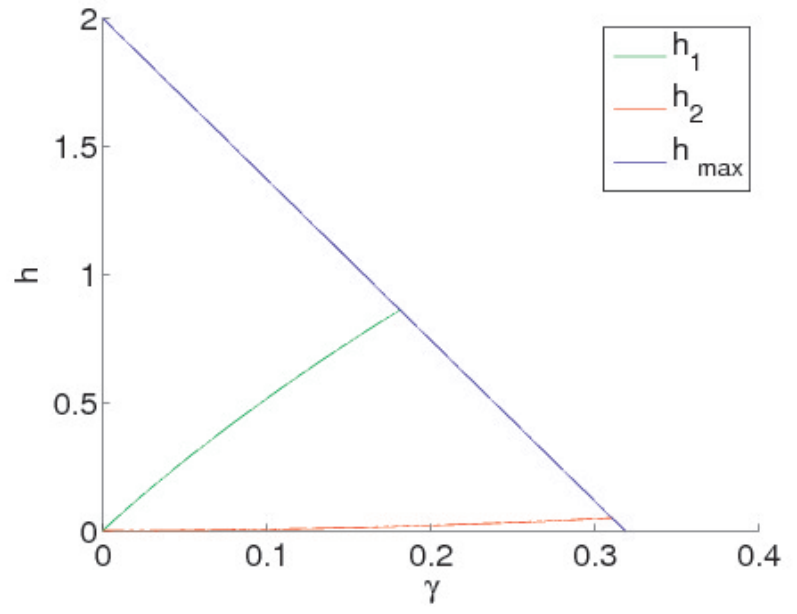

Figure 12: The extremal $h$-values $h_{1}(\gamma), h_{2}(\gamma)$ and $h_{\max }(\gamma)$.

In Figure 13, we have taken $\gamma=0.15$ and $h=\left(h_{1}+h_{\max }\right) / 2$ and have plotted all five possible pinned fluxons (i.e. all possibilities for $\left.\left(\phi_{\text {out }}, p_{\text {out }}\right)\right)$ with $\phi_{\text {in }}=\pi-\arccos (2 \pi \gamma-(1-h))$. Obviously, five more pinned fluxons with the same $\left(\phi_{\text {out }}, p_{\text {out }}\right)$ are possible with $\phi_{\text {in }}=\pi+\arccos (2 \pi \gamma-(1-h))$.

To determine the length of the inhomogeneity for the pinned fluxons, we use that on the orbits in the inhomogeneity (blue curves in the phase portrait) $\phi$ and $\phi_{x}$ are related by $\left|\phi_{x}\right|=\sqrt{2\left(H_{0}(\gamma)+h-\gamma \phi\right)}$. Integrating this ODE, taking into account the sign of $p_{\text {out }}$, we get that the length of the pinned fluxons with $p_{\text {out }}>0$ is given by

$$
2 L=\frac{\sqrt{2}}{\gamma}\left[\sqrt{H_{0}+h-\gamma \phi_{\text {in }}}-\sqrt{H_{0}+h-\gamma \phi_{\text {out }}}\right]=\frac{p_{\text {in }}-p_{\text {out }}}{\gamma}
$$

and for $p_{\text {out }}<0$, we have

$$
2 L=\frac{\sqrt{2}}{\gamma}\left[\sqrt{H_{0}+h-\gamma \phi_{\text {in }}}+\sqrt{H_{0}+h-\gamma \phi_{\text {out }}}\right]=\frac{p_{\text {in }}-p_{\text {out }}}{\gamma} .
$$



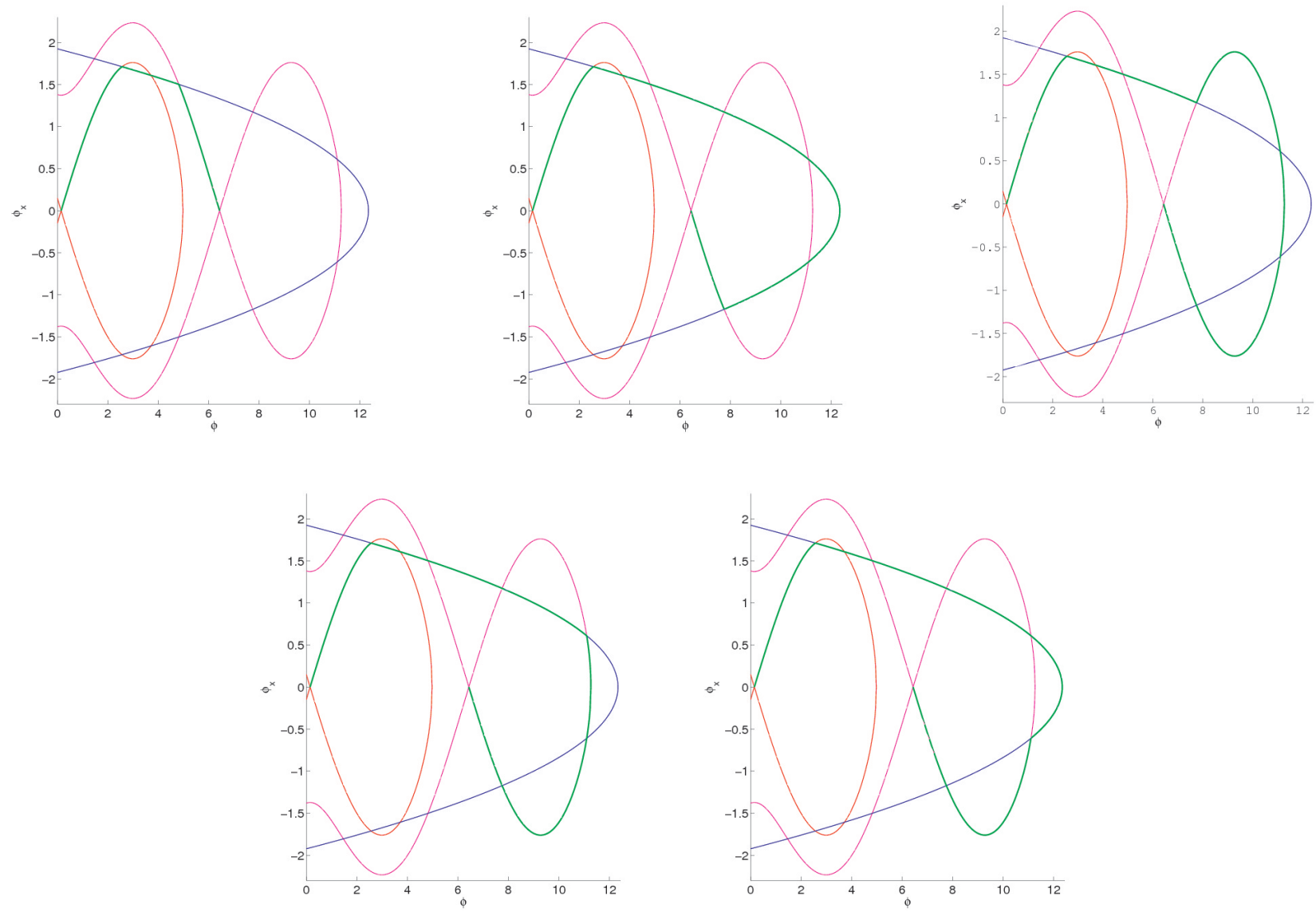

Figure 13: The five pinned fluxons with $\phi_{\text {in }}=\pi-\arccos (2 \pi \gamma-(1-h))$ for $\gamma=0.15, d=0$ and $h=\left(h_{1}+h_{\max }\right) / 2$. Note that only the pinned fluxon in the first panel is monotonic. In the $L-h$ of Figure 14, the pinned fluxons in the first two panels are on the blue curve, the third one is on the red curve and the last two are on the green curve.

These lengths are plotted in Figure 14 for $\gamma=0.15$. The blue curve is formed by the branches 1 and 2 , the red curve is branch 3 and the green curve is formed by the branches 4 and 5 . This plot shows that there is a positive minimal and maximal length for the inhomogeneity to sustain pinned fluxons. Inhomogeneities with shorter or longer lengths will not be able to sustain pinned fluxons. Figure 14 illustrates also that the maxima and minima of the possible length of the inhomogeneity are attained inside the interval $\left(0, h_{\max }\right)$, not at the endpoints. These extremal points will play an important role in the stability analysis as we will see in the next section.

Remark 2 At $\phi_{\text {out }}=2 \pi+\arcsin \gamma$, i.e $h=h_{2}$, there is no bifurcation, although a solution appears/disappears. To see that this is not a bifurcation point, we look at the disappearing solution in the limit $h \downarrow h_{2}$. For $h \downarrow h_{2}$, there is one solution with $\phi_{\text {out }} \approx 2 \pi+\arcsin \gamma$ and $p_{\text {out }}<0$ and one solution with $\phi_{\text {out }} \approx 2 \pi+\arcsin \gamma$ and $p_{\text {out }}>0$. The solution with $p_{\text {out }}<0$ remains very close to $2 \pi+\arcsin \gamma$ for $x>L$. However, the solution with $p_{\text {out }}>0$ is tracking almost all of the homoclinic connection to $2 \pi+\arcsin \gamma$. And in the limit $h \downarrow h_{2}$ this solution "splits" into the pinned fluxon with $p_{\text {out }}=0$ and a full homoclinic connection (fluxon-antifluxon pair).

In general, the derivation of the existence of the pinned fluxons shows that for fixed $\gamma>0$ and $d=0$, there will always be a strictly positive minimal and maximal length for the existence of pinned fluxons. From Figure 12, it follows that the green curve of pinned fluxons with $\phi_{\text {out }}=4 \pi-\arccos (1-h)$ is not present if $\gamma>\gamma_{1}$. Similarly if $\gamma>\gamma_{2}$, the red curve of pinned fluxons with $\phi_{\text {out }}=2 \pi+\arccos (1-h)$ 

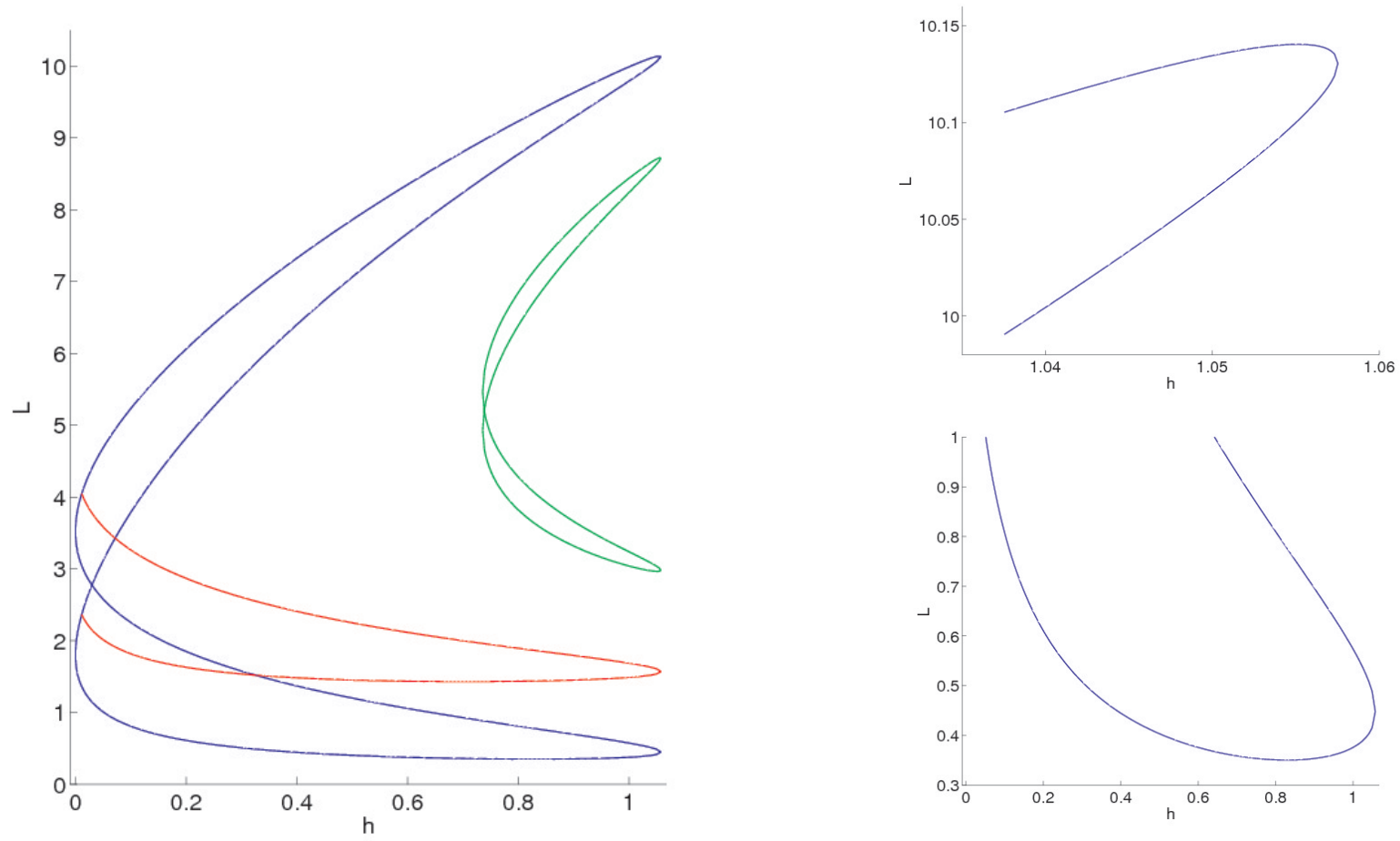

Figure 14: The lengths of the pinned fluxons for $\gamma=0.15$ and $d=0$. The lengths of the pinned fluxons with $\phi_{\text {out }}=4 \pi-\arccos (1-h)$ are plotted in green (branches 4 and 5 ), the lengths of pinned fluxons with $\phi_{\text {out }}=2 \pi+\arccos (1-h)$ and $p_{\text {out }}>0$ are in red (branch 3$)$. The lengths of the remaining pinned fluxons (branches 1 and 2) are indicated by the blue curves. The panels on the right zoom into the top and bottom and show that the minimal and maximal length are not obtained for $h_{\max }$, but a smaller value.

and $p_{\text {out }}>0$ are not present. Below we summarise the results for the existence of the pinned fluxons with an induced current:

Theorem 3 For $d=0$ and every $0<\gamma \leq \frac{1}{\pi}$, there are $L_{\min }(\gamma)$ and $L_{\max }(\gamma)$, such that for every $L \in\left(L_{\min }, L_{\max }\right)$, there are at least two pinned fluxons (at least one for $L={ }_{\min }$ or $L_{\max }$ ). Furthermore

$$
\lim _{\gamma \downarrow 0} L_{\min }(\gamma)=0, \quad \lim _{\gamma \downarrow 0} L_{\max }(\gamma)=\infty,
$$

and

$$
\lim _{\gamma \uparrow 1 / \pi} L_{\min }(\gamma)=\lim _{\gamma \uparrow 1 / \pi} L_{\max }(\gamma)=\sqrt{\frac{\pi}{2}\left(\arcsin \frac{1}{\pi}+\sqrt{\pi^{2}-1}\right)}-\sqrt{\frac{\pi}{2}\left(\arcsin \frac{1}{\pi}+\sqrt{\pi^{2}-1}-\pi\right)} \approx 1.8 .
$$

For given $L \in\left[L_{\min }, L_{\max }\right]$, the maximum possible number of simultaneously existing pinned fluxons is 6. For $\gamma>\frac{1}{\pi}$, there exist no pinned fluxons.

To relate the rich family of pinned fluxons which exists for $\gamma>0$ with the unique pinned fluxons for $\gamma=0$, we have sketched the $L$ - $h$ curves for $\gamma=0.001$ in Figure 15. The bold blue curve converges clearly to the curve in Figure 9. These are the lengths associated with the pinned fluxons with $\phi_{\text {in }}=\pi-$ $\arccos (2 \pi \gamma-(1-h))$ and $\phi_{\text {out }}=2 \pi-\arccos (1-h)$. Clearly there are some other convergent $L-h$ curves as well. The length of the blue curve associated with the pinned fluxons with $\phi_{\text {in }}=\pi+\arccos (2 \pi \gamma-(1-h))$ and $\phi_{\text {out }}=2 \pi-\arccos (1-h)$ goes to zero as expected. The convergent red and green curves can be associated with $4 \pi$-fluxons in the case $\gamma=0$. A $4 \pi$-fluxon is a connection between 0 and $4 \pi$. This is not possible without an inhomogeneity, but with an inhomogeneity such connections are possible and some are stable. There are four possible $4 \pi$-fluxons if $\gamma=0$ and the green and red curves converge to those solutions. For more details, see [18]. 


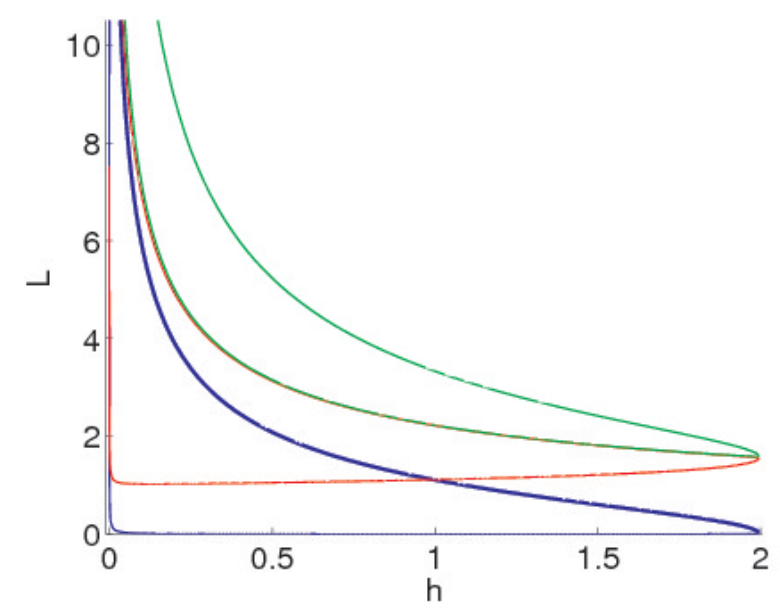

Figure 15: $L-h$ curves of the pinned fluxons for $\gamma=0.001$.

\subsection{Stability of the pinned fluxons with $d=0$}

As seen in the introduction, the stability of the pinned fluxons is determined by the eigenvalues of the linearisation operator $\mathcal{L}_{\text {pin }}$ as defined in (7). For $d=0$, the linearisation operator takes the form

$$
\mathcal{L}_{\text {pin }}(x ; L, \gamma, 0)=\left\{\begin{aligned}
D_{x x}-\cos \phi_{\text {pin }}(x ; L, \gamma, 0), & |x|>L ; \\
D_{x x}, & |x|<L .
\end{aligned}\right.
$$

where $\phi_{\text {pin }}$ is one of the pinned fluxons found in the previous section.

When there is no induced current $(\gamma=0)$, expressions for the eigenvalues of $\mathcal{L}_{\text {pin }}$ can be found explicitly. Recall that for $d=0$ and $\gamma=0$, there is a unique pinned fluxon for each length $L \geq 0$, see Lemma 1.

Lemma 4 For $\gamma=0$ and $d=0$, the linear operator $\mathcal{L}_{\text {pin }}$ associated to the unique pinned fluxon in the defect with length $L$ has a largest eigenvalue $\Lambda_{\max } \in(-1,0)$ given implicitly by the largest solution of

$$
\begin{aligned}
-\mu\left[\mu+\frac{1}{2}\right. & \left.\sqrt{2\left(1+\cos \phi_{\text {in }}\right)}\right]+\frac{1}{2}\left(1-\cos \phi_{\text {in }}\right)= \\
& -\sqrt{1-\mu^{2}}\left[\mu+\frac{1}{2} \sqrt{2\left(1+\cos \phi_{\text {in }}\right)}\right] \tan \left(\sqrt{1-\mu^{2}} \frac{\pi-\phi_{\text {in }}}{\sqrt{2\left(1-\cos \phi_{\text {in }}\right)}}\right),
\end{aligned}
$$

where $\mu=\sqrt{1+\Lambda_{\max }} \in(0,1)$ and the relation between $\phi_{\mathrm{in}}$ and $L$ is given in (10) and (11).

In Figure 16, $\Lambda_{\max }$ is sketched as function of the half-length $L$ of the pinned fluxon. The proof of Lemma 4 is quite technical; it is given in appendix A.

Remark 5 For L large (hence $\phi_{\text {in }}$ small), equation (18) has more solutions. Hence for those pinned fluxons $\mathcal{L}_{\text {pin }}$ has some smaller eigenvalues in $(-1,0)$ too.

Corollary 6 If there is no induced bias current $(\gamma=0)$ and the microresistor has $d=0$, then the unique pinned fluxon in the defect with length $L$ is linearly and nonlinearly stable. The pinned fluxon is asymptotically stable if $\alpha>0$.

Next we consider the case that there is an induced bias current $(\gamma>0)$. In the previous section we have seen that in this case the pinned fluxons come in families, characterised by the blue, red and green curves in Figure 14. Locally along those curves, we can write either $L$ as a function of $h$, or, $h$ as a function of $L$. Along those curves, we will look for changes of stability, i.e., find whether the 


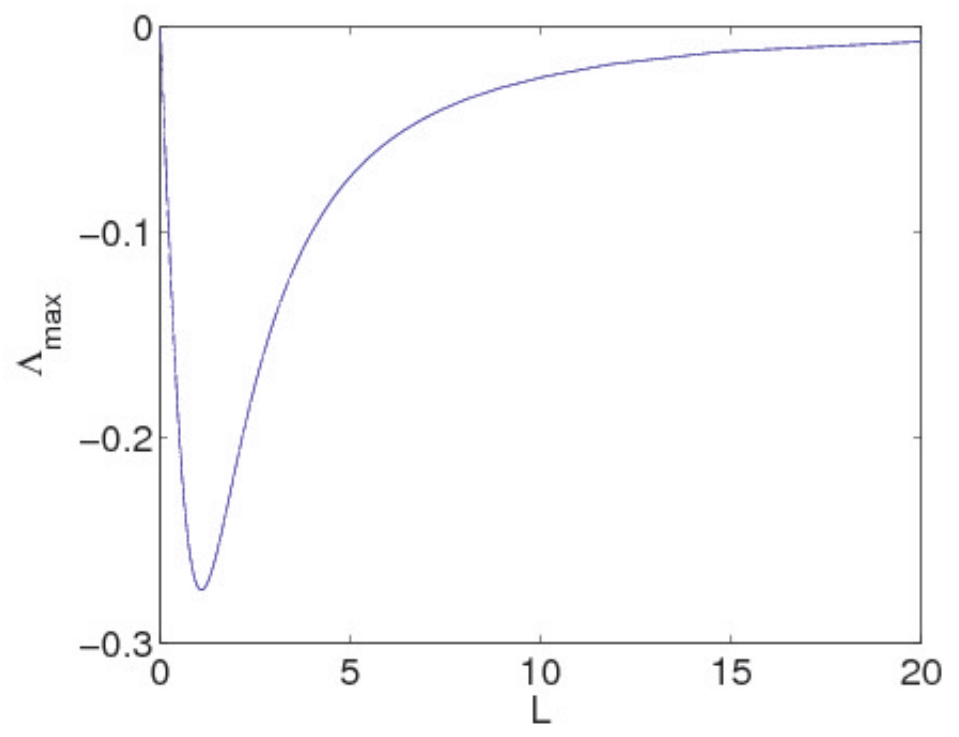

Figure 16: The largest eigenvalue of the linearised operator $\mathcal{L}_{\text {pin }}$ at $d=0$ and $\gamma=0$ as function of the half-length $L$ of the inhomogeneity.

operator $\mathcal{L}_{\text {pin }}$ has an eigenvalue 0 (recall that eigenvalues of $\mathcal{L}_{\text {pin }}$ must be real. We will show that $\mathcal{L}_{\text {pin }}$ has an eigenvalue 0 if and only if along the $L-L$ curve we have $\frac{d L}{d h}=0$ or the pinned fluxon is isolated. Isolated pinned fluxons occur when $\gamma$ is maximal, i.e., $\gamma=\frac{1}{\pi}$ or when $\gamma=\gamma_{1}$, the maximal $\gamma$-value for which pinned fluxons with $\phi_{\text {out }}=4 \pi-\arccos (2 \pi \gamma-1)$ exist. This lemma is a special case of a more general theorem presented in [19]. The proof simplifies considerably in this case.

Lemma 7 For any $\gamma \geq 0$, the linear operator $\mathcal{L}_{\text {pin }}(x ; L, \gamma, 0)$ has an eigenvalue zero if and only if

- $\frac{d L}{d h}=0$;

- or $\gamma=\frac{1}{\pi}$ (this eigenvalue zero is the largest eigenvalue);

- or $\gamma=\gamma_{1} \approx 0.18$, the solution of $\cos \phi_{\max }\left(\gamma_{1}\right)+1=2 \pi \gamma_{1}$ (see section 3.2), and $\phi_{\text {pin }}$ is such that $\phi_{\text {in }}=\pi, \phi_{\text {out }}=4 \pi-\arccos \left(2 \pi \gamma_{1}-1\right)=2 \pi+\phi_{\max }\left(\gamma_{1}\right)$ (this eigenvalue zero is not the largest eigenvalue).

Proof First we observe that differentiating (4) with respect to $x$ shows that $\phi_{\text {pin }}^{\prime}$ satisfies $\mathcal{L}_{\text {pin }} \phi_{\text {pin }}^{\prime}=0$. However, it follows immediately from (4) that $\phi_{\text {pin }}^{\prime}$ is not continuously differentiable, except when there exist $k_{ \pm} \in \mathbb{N}$ such that $\phi_{\text {in }}=k_{-} \pi$ and $\phi_{\text {out }}=k_{+} \pi$. From the existence results, it follows that this happens only if $\gamma=\frac{1}{\pi}$. In this case, there is only one pinned fluxon and the blue curve in Figure 14 has become a single point (there are no red or green curves).

In all other cases, $\phi_{\text {pin }}^{\prime} \notin C^{1}(\mathbb{R}) \supset H^{2}(\mathbb{R})$ so $\phi_{\text {pin }}^{\prime}$ is not an eigenfunction with the eigenvalue zero. However, $\phi_{\text {pin }}^{\prime}$ still plays a role in the eigenfunction related to any eigenvalue zero. Indeed, on both intervals $(\infty,-L)$ and $(L, \infty)$, the second order linear ODE $\mathcal{L}_{\text {pin }} \psi=0$ has two linearly independent solutions. As the asymptotic system is hyperbolic, one solution is exponentially decaying whilst the other is exponentially growing. Thus if the linear operator $\mathcal{L}$ has an eigenvalue zero, then the eigenfunction in the intervals $(-\infty, L)$ and $(L, \infty)$ must be a multiple of the exponentially decaying solution. As $\phi_{\text {pin }}^{\prime}$ is exponentially decaying for $|x| \rightarrow \infty$ and satisfies $\mathcal{L} \phi_{\text {pin }}^{\prime}=0$ for $|x|>L$, it follows that for any eigenvalue zero, the eigenfunction must be a multiple of $\phi_{\text {pin }}^{\prime}$ for $|x|>L$, unless $\phi_{\text {pin }}^{\prime} \equiv 0$. The case $\phi_{\text {pin }}^{\prime} \equiv 0$ happens only when $\phi_{\text {out }}=2 \pi+\arcsin \gamma$ and $x>L$. In this case, the appropriate eigenfunction is a multiple of $e^{-\sqrt[4]{1-\gamma^{2}}(x-L)}$. 
Next we look inside the inhomogeneity, i.e., $|x|<L$. The linearised problem inside the defect for an eigenvalue zero can be solved explicitly and gives an eigenfunction of the form $A+B(x+L)$, with $A$ and $B$ free parameters and $|x|<L$.

To conclude, if the linear operator $\mathcal{L}_{\text {pin }}$ has an eigenvalue zero, and $\phi_{\text {out }} \neq 2 \pi+\arcsin \gamma$ (we will consider this case later), then the eigenfunction is of the form

$$
\psi=\left\{\begin{aligned}
\phi_{\text {pin }}^{\prime}(x), & x<-L, \\
A+B(x+L), & |x|<L, \\
K \phi_{\text {pin }}^{\prime}(x), & x>L,
\end{aligned}\right.
$$

where $A, B$ and $K$ are free parameters. We have to choose the free parameters such that $\psi$ is continuously differentiable at $\pm L$. As there are only three free parameters and four matching conditions, this will give us a selection criterion on the length $L$ for which an eigenvalue zero exists. The matching conditions are

$$
A=\phi_{\text {pin }}^{\prime}\left(-L^{-}\right), \quad B=\phi_{\text {pin }}^{\prime \prime}\left(-L^{-}\right), \quad B=K \phi_{\text {pin }}^{\prime \prime}\left(L^{+}\right), \quad \text { and } \quad A+2 B L=K \phi_{\text {pin }}^{\prime}\left(L^{+}\right),
$$

where the notation $\phi_{\text {pin }}^{\prime}\left(-L^{-}\right)=\lim _{x \uparrow-L} \phi_{\text {pin }}^{\prime}(x), \phi_{\text {pin }}^{\prime}\left(L^{+}\right)=\lim _{x \downarrow L} \phi_{\text {pin }}^{\prime}(x)$, etc. Using that $p_{\text {in/out }}=$ $\phi_{\text {pin }}^{\prime}(\mp L)$ and $\gamma+\phi_{\text {pin }}^{\prime \prime}\left( \pm L^{ \pm}\right)=\sin \phi( \pm L)=\sin \phi_{\text {in/out }}$, this can be written as

$$
A=p_{\text {in }}, \quad B=\sin \phi_{\text {in }}-\gamma, \quad B=K\left(\sin \phi_{\text {out }}-\gamma\right), \quad \text { and } \quad A+2 B L=K p_{\text {out }} .
$$

Equations (16) and (17) show that $L=\frac{p_{\text {in }}-p_{\text {out }}}{2 \gamma}$, hence the parameters are given by

$$
A=p_{\text {in }}, \quad B=\sin \phi_{\text {in }}-\gamma, \quad \text { and } \quad K\left(\sin \phi_{\text {out }}-\gamma\right)=\sin \phi_{\text {in }}-\gamma
$$

and the compatibility condition on $L$, or equivalently $h$, is

$$
0=p_{\text {in }} \sin \phi_{\text {in }}\left(\sin \phi_{\text {out }}-\gamma\right)-p_{\text {out }} \sin \phi_{\text {out }}\left(\sin \phi_{\text {in }}-\gamma\right) .
$$

To derive this expression, we have multiplied the remaining equation $\left[A+2 B L=K p_{\text {out }}\right]$ with $\gamma\left(\sin \phi_{\text {out }}-\right.$ $\gamma$ ). This term would be zero if $\sin \phi_{\text {out }}=\gamma$, hence $\phi_{\text {out }}=2 \pi+\arcsin \gamma$ but this case is not considered now.

For completeness, we also consider the case where we assume that the eigenfunction vanishes for $x<-L$. If this is the case, then matching at $x=-L$ gives immediately that $A=0=B$. Thus this leads to a non-trivial eigenfunction only if $\phi_{\text {pin }}^{\prime}(L)=0=\lim _{x \downarrow L} \phi_{\text {pin }}^{\prime \prime}(x)$. In other words, when $\phi_{\text {pin }}$ is a fixed point for $x>L$. This happens only if $\phi_{\text {out }}=2 \pi+\arcsin \gamma$. This case we will be considered later.

Next we link the expression (19) to the derivative of $L$ with respect to $h$. As $L=\frac{p_{\text {in }}-p_{\text {out }}}{2 \gamma}$, the derivatives of $p_{\text {in }}$ and $p_{\text {out }}$ are needed. Differentiating (14) and (15), we get

$$
p_{\text {in }} \frac{d p_{\text {in }}}{d h}=1-\gamma \phi_{\text {in }}^{\prime}(h), \quad \sin \phi_{\text {in }} \frac{d \phi_{\text {in }}}{d h}=1 \quad \text { and } \quad p_{\text {out }} \frac{d p_{\text {out }}}{d h}=1-\gamma \phi_{\text {out }}^{\prime}(h), \quad \sin \phi_{\text {out }} \frac{d \phi_{\text {out }}}{d h}=1 .
$$

Thus differentiating $L=\frac{p_{\text {in }}-p_{\text {out }}}{2 \gamma}$ gives that

$$
p_{\text {in }} \sin \phi_{\text {in }} p_{\text {out }} \sin \phi_{\text {out }} \frac{d L}{d h}=\frac{1}{2 \gamma}\left[p_{\text {out }} \sin \phi_{\text {out }}\left(\sin \phi_{\text {in }}-\gamma\right)-p_{\text {in }} \sin \phi_{\text {in }}\left(\sin \phi_{\text {out }}-\gamma\right)\right]
$$

So we have shown that if $\phi_{\text {out }} \neq 2 \pi+\arcsin \gamma$ and the operator $\mathcal{L}_{\text {pin }}$ has an eigenvalue zero, then either $\frac{d L}{d h}(h, \gamma)=0$ or $p_{\text {in }} \sin \phi_{\text {in }} p_{\text {out }} \sin \phi_{\text {out }}=0$. Considering $p_{\text {in }} \sin \phi_{\text {in }} p_{\text {out }} \sin \phi_{\text {out }}=0$ in more detail, we get:

- $\sin \phi_{\text {out }}=0$ would mean that $\phi_{\text {out }}=2 \pi$. Going back to the compatibility condition (19), this implies that $\gamma p_{\text {in }} \sin \phi_{\text {in }}=0$, which only happens if also $\sin \phi_{\text {in }}=0$ or $p_{\text {in }}=0$. In the existence section we have seen $p_{\text {in }}>0$, hence $\gamma p_{\text {in }} \sin \phi_{\text {in }}=0$ can only happen if $\phi_{\text {in }}=\pi$, hence if $\gamma=\frac{1}{\pi}$; 
- $\sin \phi_{\text {in }}=0$ implies that $\phi_{\text {in }}=\pi$. Going back to the compatibility condition (19), this implies that $\gamma p_{\text {out }} \sin \phi_{\text {out }}=0$, which only happens if also $\sin \phi_{\text {out }}=0$ or $p_{\text {out }}=0$. Hence either $\gamma=\frac{1}{\pi}$ or $\gamma=\gamma_{1}$, as the case $\phi_{\text {out }}=2 \pi+\arcsin \gamma$ is excluded at this moment;

- $p_{\text {in }} \neq 0$ as we have seen before;

- $p_{\text {out }}=0$ happens if $\phi_{\text {out }}=2 \pi+\arcsin \gamma$ or $\phi_{\text {out }}=2 \pi+\phi_{\max }(\gamma)$. Going back to the compatibility condition (19), this implies that $p_{\text {in }} \sin \phi_{\text {in }}\left(\sin \phi_{\text {out }}-\gamma\right)=0$. Since $\pi-\arcsin \gamma<\phi_{\max }(\gamma)<2 \pi$, this implies this only happens if $\sin \phi_{\text {in }}=0$, which case is considered before.

So altogether we have if $\phi_{\text {out }} \neq 2 \pi+\arcsin \gamma$ and the operator $\mathcal{L}_{\text {pin }}$ has an eigenvalue zero, then either

- $\frac{d L}{d h}(h, \gamma)=0$ or

- $\phi_{\text {in }}=\pi$ and $\phi_{\text {out }}=2 \pi$, which only happens when $\gamma=\frac{1}{\pi}$. The eigenfunction in this case is $\phi_{\text {pin }}^{\prime}$, which does not have any zeros, hence the eigenvalue zero is the largest eigenvalue.

- $\phi_{\text {in }}=\pi$ and $\phi_{\text {out }}=2 \pi+\phi_{\max }(\gamma)$ (i.e. $p_{\text {out }}=0$ ), which only happens if $\gamma=\gamma_{1}$. In this case the eigenfunction is $\phi_{\text {pin }}^{\prime}$ for $x<L$ and $\frac{\gamma_{1}}{\gamma_{1}-\sin \phi_{\max }\left(\gamma_{1}\right)} \phi_{\text {pin }}^{\prime}$ for $x>L$. This eigenfunction has a zero at $x=L$, hence the eigenvalue zero is not the largest eigenvalue. Note that when $\gamma=\gamma_{1}$ the green $L(h)$ curve in Figure 14 has degenerated to an isolated point related to the pinned fluxon $\phi_{\text {pin }}$ considered in this case.

To show that the converse is true, we look at the three cases $\frac{d L}{d h}(h, \gamma)=0, \gamma=\frac{1}{\pi}$ and $\gamma=\gamma_{1}$ and $\left(\phi_{\text {out }}, p_{\text {out }}\right)=\left(2 \pi+\phi_{\max }, 0\right)$. It is straightforward to verify that the eigenfunctions as described earlier can be constructed in those cases.

Finally we look at the case $\phi_{\text {out }}=2 \pi+\arcsin \gamma$. In this case $\gamma \leq \frac{4 \pi}{1+4 \pi^{2}}$ and $h=h_{2}=1-\sqrt{1-\gamma^{2}}$. Furthermore, the pinned fluxons satisfies $\phi_{\text {pin }}^{\prime} \equiv 0$ for $x>L$. In this case, the general form of an eigenfunction for an eigenvalue zero is

$$
\psi=\left\{\begin{aligned}
\phi_{\text {pin }}^{\prime}(x), & x<-L, \\
A+B(x+L), & |x|<L, \\
K e^{-\sqrt[4]{1-\gamma^{2}}(x-L)}, & x>L,
\end{aligned}\right.
$$

where $A, B$ and $K$ are free parameters. We have to choose the free parameters such that $\psi$ is continuously differentiable at $= \pm L$, i.e.

$$
A=\phi_{\text {pin }}^{\prime}\left(-L^{-}\right), \quad B=\phi_{\text {pin }}^{\prime \prime}\left(-L^{-}\right), \quad K=A+2 B L, \quad \text { and } \quad B=-K \sqrt[4]{1-\gamma^{2}} .
$$

As $L=\frac{p_{\text {in }}-p_{\text {out }}}{2 \gamma}=\frac{p_{\text {in }}}{2 \gamma}$, this implies that $A=p_{\text {in }}, B=\sin \phi_{\text {in }}-\gamma$ and $K=\frac{p_{\text {in }} \sin \phi_{\text {in }}}{\gamma}$, with the matching condition

$$
\gamma\left(\sin \phi_{\text {in }}-\gamma\right)=-\sqrt[4]{1-\gamma^{2}} \sin \phi_{\text {in }} p_{\text {in }} .
$$

If $\phi_{\text {in }}=\pi+\arccos \left(2 \pi \gamma-\sqrt{1-\gamma^{2}}\right)$, then $\sin \phi_{\text {in }}<0$ and (21) cannot be satisfied as $p_{\text {in }}>0$ and $\gamma>0$. If $\phi_{\text {in }}=\pi-\arccos \left(2 \pi \gamma-\sqrt{1-\gamma^{2}}\right)$, then the phase portrait in the existence section shows that $\sin \phi_{\text {in }}>\gamma$, thus $\left(\sin \phi_{\text {in }}-\gamma\right)>0$ and again (21) cannot be satisfied. Thus no eigenvalue zero can occur at $\phi_{\text {out }}=2 \pi+\arcsin \gamma$.

Lemma 7 allows us to conclude the stability of pinned fluxons.

Theorem 8 For $d=0$, every $0<\gamma \leq \frac{1}{\pi}$, and every $L \in\left[L_{\min }(\gamma), L_{\max }(\gamma)\right]$, there is exactly one stable pinned fluxon. This pinned fluxon is linearly and nonlinearly stable (and asymptotically stable for $\alpha>0)$. For $L$ sufficiently large $\left(L>\sqrt{\frac{\pi+\arcsin \gamma+\arccos \left(2 \pi \gamma-\sqrt{1-\gamma^{2}}\right)}{2 \gamma}}\right)$, the stable pinned fluxons are non-monotonic. 

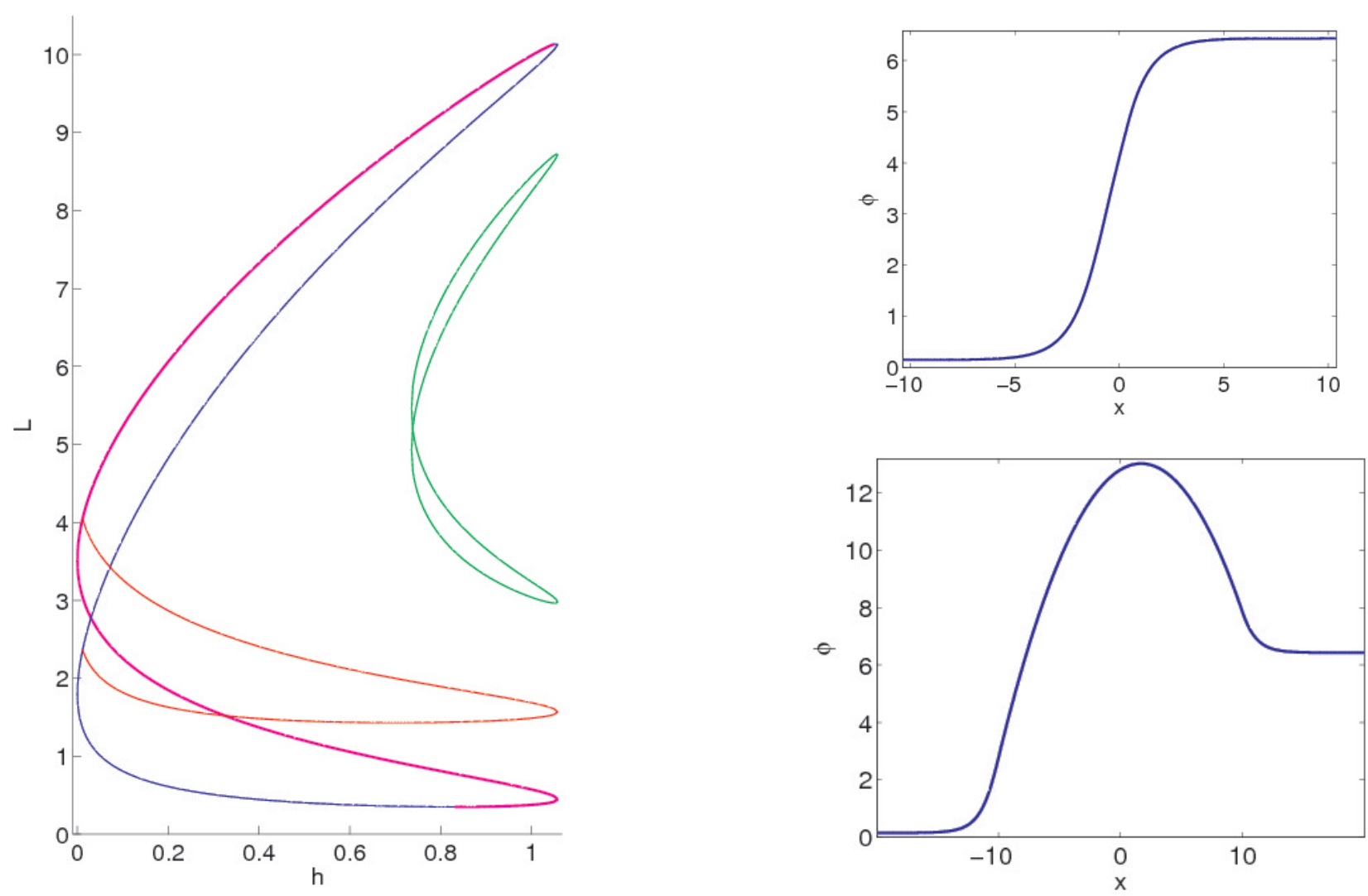

Figure 17: Stability for $d=0$ and $\gamma=0.15$. The bold magenta curve represents stable solutions, all other solutions are unstable. On the right there is an example of a stable monotonic pinned fluxon (at $L=0.38$ ) and a stable non-monotonic one (at $L=10$ ), Both stable pinned fluxons have $h=1$, i.e. they are near minimal respectively maximal length, which are at $L_{\min }=0.35$ and $L_{\max }=10.13$.

See Figure 17 for an illustration of this theorem.

Proof If $\gamma=\frac{1}{\pi}$, then only the inhomogeneity with half-length exactly $L=\sqrt{\frac{\pi}{2}\left(\arcsin \frac{1}{\pi}+\sqrt{\pi^{2}-1}\right)}-$ $\sqrt{\frac{\pi}{2}\left(\arcsin \frac{1}{\pi}+\sqrt{\pi^{2}-1}-\pi\right)} \approx 1.8$ has a pinned fluxon. From Lemma 7, it follows that the linearisation for this pinned fluxon has a largest eigenvalue 0 , so this pinned fluxon is linearly stable.

In Corollary 6, we have seen that the unique pinned fluxons for $\gamma=0$ are stable.

If $0<\gamma<\frac{1}{\pi}$, then there are at least two pinned fluxons if $L \in\left(L_{\min }, L_{\max }\right)$, see Theorem 3. As seen before, the $L$ - $h$ curves for the pinned fluxons form three isolated curves: $\phi_{\text {out }}=4 \pi-\arccos (1-h)$ (green curve), the (red) curve of pinned fluxons with $\phi_{\text {out }}=2 \pi+\arccos (1-h)$ and $p_{\text {out }}>0$ (exists for $h>h_{2}$ ), and the other pinned fluxons (blue curve). The colour coding refers to Figures 14 and 17 . The fluxons on the blue curve exist for all $0 \leq \gamma \leq \frac{1}{\pi}$; the existence of the other curves depends on the value of $\gamma$.

The linearisation about the pinned fluxon at the minimum on the red curve has an eigenvalue zero. At this point, the associated eigenfunction is a multiple of $\phi_{\text {pin }}^{\prime}$ for $x>L$. On the red curve, $p_{\text {out }}>0$ and $\phi_{\text {pin }}^{\prime}(x)<0$ for $x$ large. Thus this eigenfunction has a zero. Using Sturm-Liouville theory, we can conclude that the eigenvalue zero is not the largest eigenvalue. As there is only one fluxon with $\frac{d L}{d h}=0$ on the red curves, all pinned fluxons on the red curve are linearly unstable.

Similarly, the minimum and maximum on the green curve are associated with pinned fluxons whose linearisation has an eigenvalue zero. Again, the associated eigenfunction for $x>L$ is a multiple of 
$\phi_{\text {pin }}^{\prime}$. As for the red curve, at the minimum we have $p_{\text {out }}>0$ and $\phi_{\text {pin }}^{\prime}(x)<0$ for $x$ large. Thus this eigenfunction has a zero and we can conclude that the eigenvalue zero is not the largest eigenvalue. The green curve is a closed curve with only two points with $\frac{d L}{d h}=0$, so the eigenvalue zero at the maximum cannot be the largest eigenvalue either. So we can conclude that all pinned fluxons on the green curve are linearly unstable.

Finally we consider the blue curve. We use the stability of the pinned fluxons at $d=0, \gamma=0$ to get a conclusion about the stability of the pinned fluxons on this curve. The solutions that can be continued to $\gamma=0$ are the connections between $\phi_{\text {in }}=\pi-\arccos (2 \pi \gamma-1+h)$ and $\phi_{\text {out }}=2 \pi-\arccos (1-h)$. Hence those solutions are stable. Now using that zero eigenvalues can only occur if $L(h)$ has a critical point, the blue curve can be divided in stable and unstable solutions. The stable solutions are the part of the curve $L(h)$ curve between the minimum and maximum that contains the pinned fluxons with $\phi_{\text {in }}=\pi-\arccos (2 \pi \gamma-1+h)$ and $\phi_{\text {out }}=2 \pi-\arccos (1-h)$. The pinned fluxons in the other part are unstable as the zero eigenvalue is simple. It can be verified that the eigenfunctions related to the zero eigenvalues on this curve do not have any zeroes indeed.

So altogether we can conclude that for each length there is exactly one stable and at least one unstable solution. The stable fluxons are non-monotonic if $L$ is larger than the length of the fluxon at $h=h_{2}(\gamma)=1-\sqrt{1-\gamma^{2}}$ with $\phi_{\text {in }}=\pi-\arccos \left(2 \pi \gamma-\sqrt{1-\gamma^{2}}\right)$ and $\phi_{\text {out }}=2 \pi+\arcsin \gamma$, hence $L>\sqrt{\frac{\pi+\arcsin \gamma+\arccos \left(2 \pi \gamma-\sqrt{1-\gamma^{2}}\right)}{2 \gamma}}$.

\section{General case $(d>0)$}

After analysing the existence and stability of pinned fluxons in microresisors with the $d=0$ in full detail, in this section we will sketch the existence and stability of the pinned fluxons for a general microresistor or microresonator.

\subsection{Microresistors $(0<d<1)$}

The existence of pinned fluxons for $0<d<1$ follows from similar arguments as for the case $d=0$. Using the matching of appropriate solutions in the phase planes again, it can be shown that pinned fluxons exist for $0 \leq \gamma \leq \frac{1-d}{\pi}$. The Hamiltonian dynamics in the inhomogeneity satisfies the relation

$$
\frac{1}{2} \phi_{x}^{2}-d(1-\cos \phi)+\gamma \phi=H_{0}(\gamma)+h,
$$

where $h$ is a parameter for the value of the Hamiltonian as before. The case $\gamma=0$ (no induced current) is more or less identical to before, with a unique pinned fluxon for any $L>0$. For $\gamma>0$, a similar calculation as in the case $d=0$ shows that there are two possible entry angles:

$$
\phi_{\text {in }}=\pi-\arccos \left(\frac{2 \pi \gamma-(1-d-h)}{1-d}\right) \quad \text { or } \quad \phi_{\text {in }}=\pi+\arccos \left(\frac{2 \pi \gamma-(1-d-h)}{1-d}\right)
$$

and up to three possible exit angles:

$$
\phi_{\text {out }}=2 \pi-\arccos \left(\frac{1-d-h}{1-d}\right), \quad \phi_{\text {out }}=2 \pi+\arccos \left(\frac{1-d-h}{1-d}\right), \quad \text { or } \quad \phi_{\text {out }}=4 \pi-\arccos \left(\frac{1-d-h}{1-d}\right),
$$

with $0 \leq h \leq 2(1-d-\pi \gamma)$. If $\gamma>d>0$ (i.e., $d$ is sufficiently close to zero), then there is still a minimal length $L_{\min }(\gamma)>0$ and a maximal length $L_{\max }(\gamma)$ for the inhomogeneity at which pinned fluxons can exist. However, if $\gamma$ is less than $d(0<\gamma \leq d)$, then there is no upper bound on the possible length of the inhomogeneity anymore, i.e., $L_{\max }=\infty$. This new phenomenon appears for $\gamma / d \leq 1$, due to the fact that now the dynamics in the inhomogeneity have fixed points at $(\phi, p)=(2 k \pi+\arcsin (\gamma / d), 0)$, $k \in \mathbb{Z}$. If $h$ corresponds to an orbit which contains such a fixed point, then the length of an orbit with $p_{\text {out }}<0$ goes to infinity. To illustrate this, in Figure 18, we have sketched the phase portraits for $d=0.2$ and $\gamma=0.15<d$ and $\gamma=0.22>d$. 

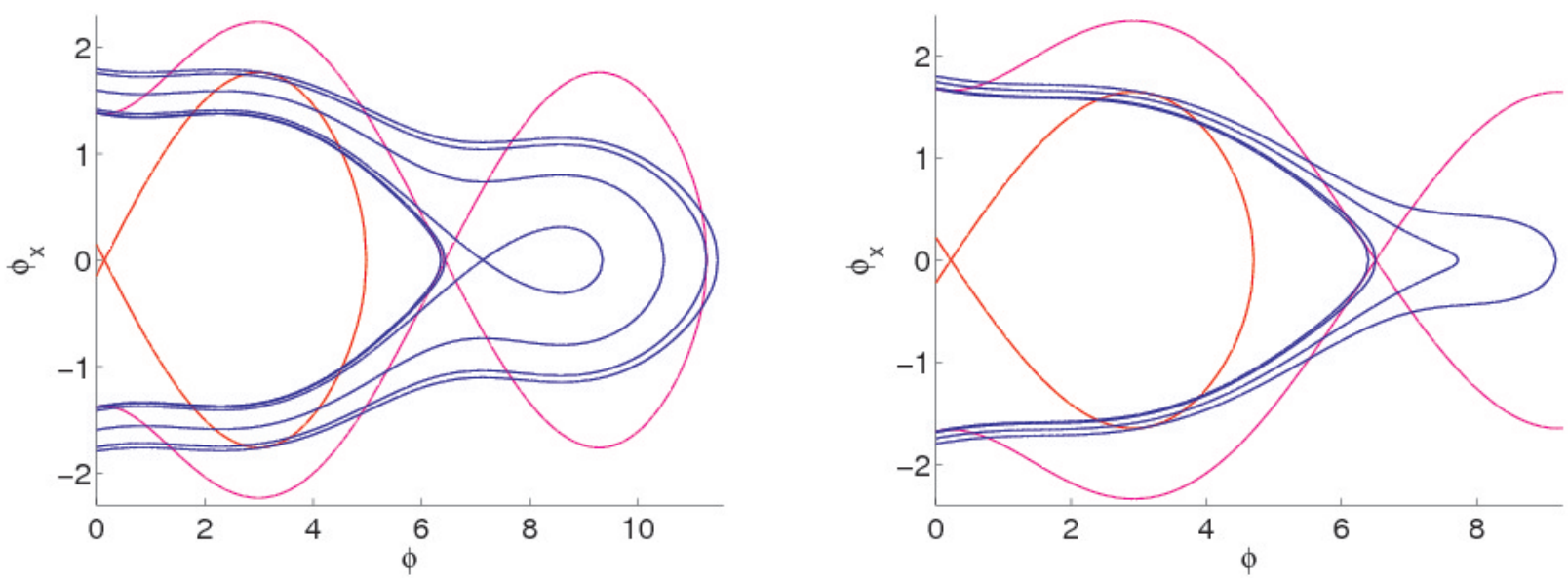

Figure 18: Phase portrait at $d=0.2$ and $\gamma=0.15$ (left) and $\gamma=0.22$ (right). Note that in the left graph, the third blue orbit has a fixed point. So the pinned fluxon with $\phi_{\text {out }}=2 \pi+\arccos \left(\frac{1-d-h}{1-d}\right)$ and $p_{\text {out }}<0$ does not exist for this $h$-value. Nearby pinned fluxons will be in a defect with a length that goes to infinity. In the right graph, there are no fixed points anymore as $\frac{\gamma}{d}>1$. Thus the defect lengths for which pinned fluxons exist are bounded.

As before, the length of the inhomogeneity for the pinned fluxons parametrised with $h$ can be determined by using the relation $\left|\phi_{x}\right|=\sqrt{2\left(H_{0}(\gamma)+h+d(1-\cos \phi)-\gamma \phi\right)}$ and integrating the ODE, taking care of the sign of $\phi_{x}$. The resulting integrals cannot be expressed analytically in elementary functions anymore, but they can be evaluated numerically. To illustrate this, we have determined the $L$ - $h$ curves as function of $h$ for $d=0.2$ and $\gamma=0.15(\gamma<d)$ and $\gamma=0.22(\gamma>d)$. The $L$ - $h$ curves are presented in Figure 19. Note the unbounded length curve for $\gamma=0.15$.
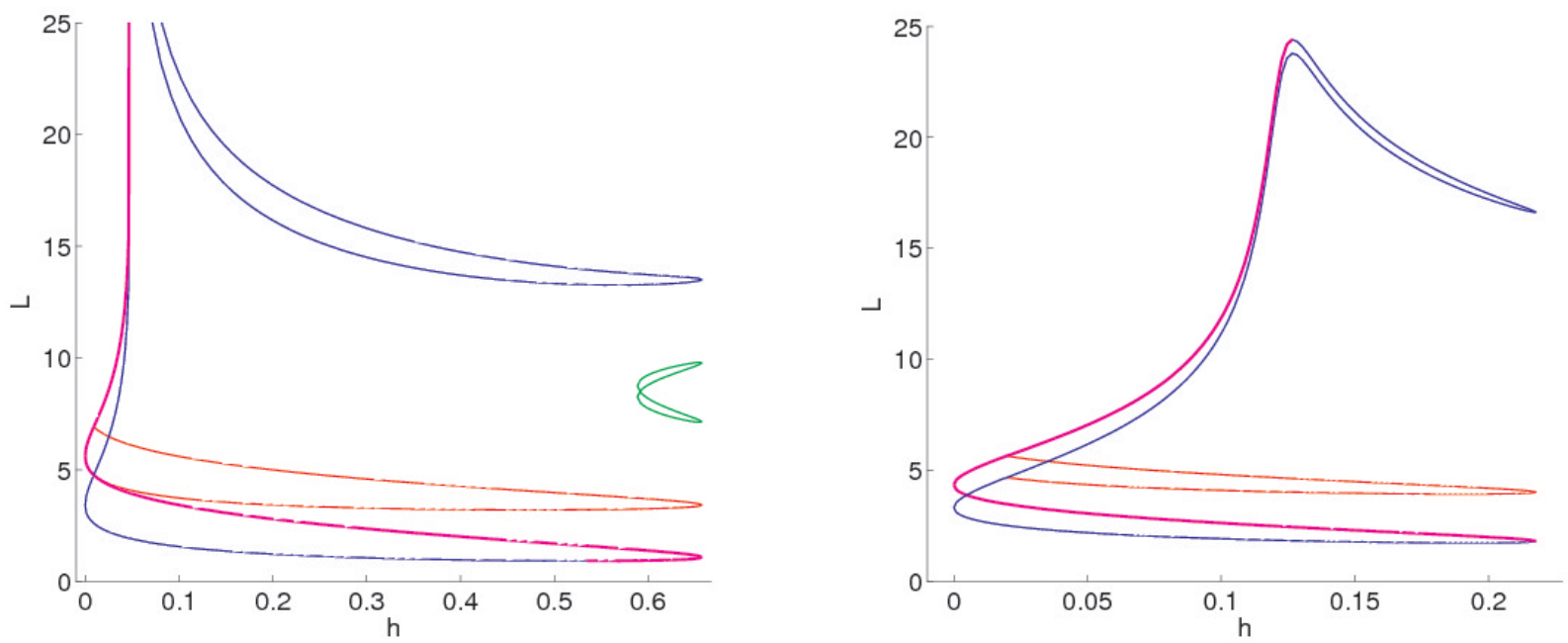

Figure 19: $L$ - $h$ curves for $d=0.2$ and $\gamma=0.15$ (left) and $\gamma=0.22$ (right). For $\gamma=0.15$, the $L$ - $h$ curves are unbounded as $\gamma$ is less than $d$. The color coding is as before, hence the bold magenta curve correspond to the stable fluxons.

In the following theorem, we summarise the existence of pinned fluxons for $0<d<1$ and give their stability.

Theorem 9 For $0<d<1$ and

- $\gamma=0$, there is a unique stable pinned fluxon for each $L \geq 0$; 
- $0<\gamma \leq \min \left(d, \frac{1-d}{\pi}\right)$, there is a minimal length $L_{\min }(\gamma)>0$ such that for all $L>L_{\min }$ there exists at least two pinned fluxons (one for $L=L_{\min }$ ). For each $L \geq L_{\min }$, there is exactly one stable pinned fluxon;

- $d<\gamma \leq \frac{1-d}{\pi}$, there are minimal and maximal lengths, $L_{\min }(\gamma)>0$ respectively $L_{\max }(\gamma)$ such that for all $L_{\min }<L<L_{\max }$ there exists at least two pinned fluxons, one pinned fluxon if $L$ is maximal or minimal, and no pinned fluxons exist for other lengths. For each $L_{\min } \leq L \leq L_{\max }$, there is exactly one stable pinned fluxon;

- for $\gamma>\frac{1-d}{\pi}$, there exist no pinned fluxons.

Note that the third case will be relevant only if $0<d<\frac{1}{\pi+1}$.

To prove the stability result for the pinned fluxons, we will use Theorem 3.1 from [19]. In [19], the stability of fronts or solitary waves in a wave equation with an inhomogeneous nonlinearity is considered. It links the existence of an eigenvalue zero of the linearisation with critical points of the $L-h$ curve. The proof has similarities with the proof of the case $d=0$ in Lemma 7 , but several extra issues have to be overcome. Theorem 3.1 of [19], applied to our pinned fluxons for $0<d<1$, leads to the following lemma, which is very similar to Lemma 7 which holds for the microresistor with $d=0$.

Lemma 10 If $0<d<1$, then the linear operator $\mathcal{L}_{\text {pin }}(x ; L, \gamma, d)$ has an eigenvalue zero if and only if

- $\frac{d L}{d h}=0$;

- or $\gamma=\frac{1-d}{\pi}$ (this eigenvalue zero is the largest eigenvalue);

- or $\gamma$ is such that it solves $(1-d)\left(\cos \phi_{\max }(\gamma)+1\right)=2 \pi \gamma$ and the pinned fluxon is such that $\phi_{\text {in }}=2 \pi+\phi_{\max }(\gamma)$ (this eigenvalue zero is not the largest eigenvalue).

The verification of Lemma 10 can be found in $[19, \S 3.5]$. As far as the special cases in this lemma is concerned, if $\gamma=\frac{1-d}{\pi}$ or $\gamma$ is such that it solves $(1-d)\left(\cos \left(\phi_{\max }(\gamma)+1\right)=2 \pi \gamma\right.$ and the pinned fluxon is such that $\phi_{\text {in }}=2 \pi+\phi_{\max }(\gamma)$, then the pinned fluxon under consideration corresponds an isolated "green" point and $\frac{d L}{d h}$ does not exist. In the case of $\gamma=\frac{1-d}{\pi}$, there is exactly one value of the length $L$ for which there exists a pinned fluxon. In the other case, there are more pinned fluxons, but on other branches. In the case of an isolated pinned fluxon, either the derivative of the pinned fluxon is an eigenfunction with the eigenvalue zero or a combination of multiples of the derivative of the pinned fluxon is an eigenfunction.

The stability result of Theorem 9 follows by combining Lemmas 4 and 10 .

Proof of Theorem 9 The existence is described at the first part of this section, in this proof we focus on the stability. For $0 \leq d<1$ and $\gamma=0$, there is a unique pinned fluxon for each length $L$. It is straightforward to show that for each $0 \leq d<1$, the length function $L(h)$ is monotonic decreasing in $h$. Thus $\frac{d L}{d h} \neq 0$ and none of the pinned fluxons has an eigenvalue zero. As all pinned fluxons are nonlinearly stable for $d=0$ (Lemma 4 ) and no change of stability can happen, all pinned fluxons with $\gamma=0$ are nonlinearly stable for all $0 \leq d<1$.

If $0<d<1$ and $0<\gamma<\frac{1-d}{\pi}$, then the $L$ - $h$ curve follows as a smooth deformation from the curve for $d=0$. And the unique stable pinned fluxon for each length follows.

If $0<d<1$ and $\gamma=\frac{1-d}{\pi}$, then the pinned fluxon is an isolated point and Lemma 10 gives that it is stable. 


\subsection{Microresonator $(d>1)$}

The existence results of pinned fluxons for $d>1$ are slightly different from the ones for $d<1$. The main difference is the type of solutions used in the inhomogeneous system. For $d<1$, we used solutions that were part of unbounded orbits or homo/heteroclinic orbits in the phase plane. For $d>1$, we have to use periodic orbits. The most simple way to understand this crucial difference between the microresistor and the microresonator case is to consider the phase portraits without applied bias current $(\gamma=0)-$ see Figure 20. When $d<1$, respectively $d>1$, the (red) heteroclinic orbit of the system outside the

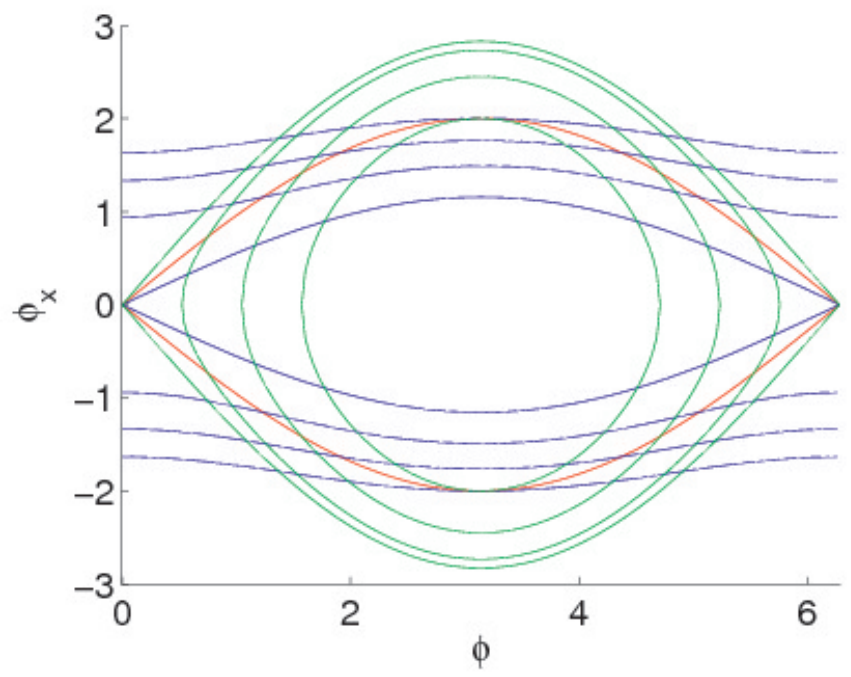

Figure 20: Phase portraits at $\gamma=0$ for various values of $d$. The red curve is the heteroclinic connection at $d=1$. The blue curves are orbits for $d=\frac{1}{2}$ and the green ones are orbits for $d=2$.

inhomogeneity is outside, resp. inside, the (blue resp. green) heteroclinic orbit of the system inside the inhomogeneity - see Figure 20. As a consequence, a pinned defect can only be constructed with (unbounded) orbits that are outside the (blue)inhomogeneous heteroclinic orbit in the microresistor case, while one has to use bounded, periodic orbits in microresonator case - see the green lines in Figure 20.

One consequence is that if one solution for a inhomogeneity of a certain length exists, then there are also solutions for inhomogeneities with lengths that are this length plus a multiple of the length of the periodic orbit. This implies that the number of pinned fluxons for a defect of length $L$ may grow without bound as $L$ increases - which is very different from the microresistor $(d<1)$. We will focus on the existence of solutions which use less than a full periodic orbit as the other ones follow immediately from this.

Using similar techniques as in the previous sections, it can be shown that if $\widehat{d}$ is the solution of $-\frac{5 \pi}{2}+\arcsin \frac{1}{d}+\sqrt{d^{2}-1}+d-1=0,(\widehat{d} \approx 4.37)$, then for $d>\widehat{d}$, pinned fluxons exist for any $0 \leq \gamma \leq 1$. If $d \leq \widehat{d}$, then pinned fluxons exist for $0 \leq \gamma<\gamma_{\max }$, where $\gamma_{\max }(d)$ is the (implicit) solution of $-2 \pi \gamma-\gamma\left(\arcsin \gamma-\arcsin \frac{\gamma}{d}\right)+\sqrt{d^{2}-\gamma^{2}}-\sqrt{1-\gamma^{2}}+(d-1)=0$.

For illustration, phase portraits for $d=4$ and various values of $\gamma$ are sketched in Figure 21. This illustrates that the solutions used in the inhomogeneous system (blue lines) are all part of a periodic orbit. Note that for $\gamma>0$ both unstable manifolds of $\arcsin \gamma$ and only the unbounded stable manifold of $2 \pi+\arcsin \gamma$ are used as opposed to the microresistor case where only the bounded unstable manifold of $\arcsin \gamma$ and both stable manifolds of $2 \pi+\arcsin \gamma$ are used.

As before, the dynamics in the inhomogeneity satisfies the relation

$$
\frac{1}{2} \phi_{x}^{2}-d(1-\cos \phi)+\gamma \phi=H_{0}(\gamma)+h,
$$



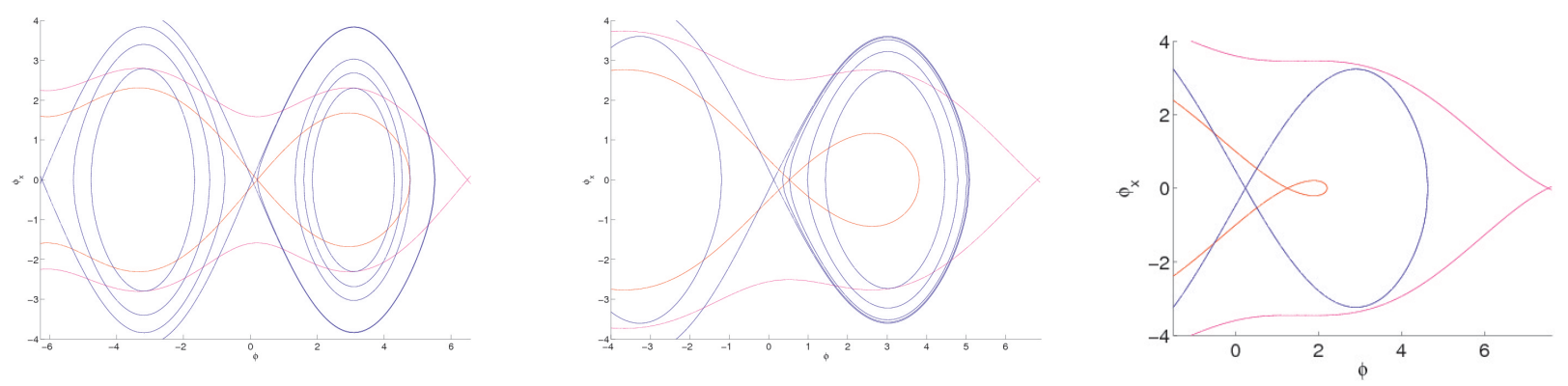

Figure 21: Phase portrait at $d=4$ and $\gamma=0.2$ (left), $\gamma=0.5$ (middle) and $\gamma=0.95$ (right). As before, the red curves are the unstable manifolds to $\arcsin \gamma$ and the magenta ones are the stable manifolds to $2 \pi+\arcsin \gamma$. The blue curves are orbits inside the inhomogeneity. The inner blue curve with angles between 0 and $2 \pi$ is the curve with the minimal $h$-value for which pinned fluxons exist. The blue curves can continue to relate to pinned fluxons up to (but not including) the blue homoclinic connection to $\arcsin \left(\frac{\gamma}{d}\right)$. Some of the periodic orbits with negative angles will also play a role in the construction of the pinned fluxons. If $\gamma=0.95>\gamma_{\max }(d) \approx 0.9$ (right plot), the blue homoclinic orbit (that encloses the red limit state) does not intersect the magenta stable manifold; illustrating that there cannot be pinned fluxons for $\gamma>\gamma_{\max }(d)$.

where $h$ is a parameter for the value of the Hamiltonian. Again it can be shown that the entry and exit angles satisfy

$$
\cos \phi_{\text {in }}=\frac{2 \pi \gamma+d-1+h}{d-1} \text { and } \quad \cos \phi_{\text {out }}=\frac{d-1+h}{d-1}
$$

where now $-2(d-1) \leq h<h_{\max }$. Here $h_{\max }$ corresponds to the $h$-value of the orbit homoclinic to $\arcsin \frac{\gamma}{d}$ in the inhomogeneous system; it can be shown that $h_{\max }<0$. As we use periodic orbits inside the inhomogeneity, the entry and exit angles will differ by less than $2 \pi$. For any $h$ value in $\left[-2(d-1), h_{\max }\right)$, there will be pinned fluxons with entry angles between $\arcsin \frac{\gamma}{d}$ and $2 \pi$. For $\gamma$ small relative to $d$, entry angles less than $\arcsin \frac{\gamma}{d}$ are also possible and they can be related to smaller (more negative) $h$ values. The $p$-value for the exit points is always positive, while the entry points can have both positive and negative $p$-values if the entry angle is larger than $\arcsin \frac{\gamma}{d}$. The pinned fluxons with entry angles less than $\arcsin \gamma$ have only negative $p_{\text {in }}$-values and hence those pinned fluxons are non-monotonic and "dip down".

For $\gamma=0$, at least one pinned fluxon exists for each $L \geq 0$. If $L$ is sufficiently large, there will be more pinned fluxons. This is different to the case with $d<1$, where for $\gamma=0$, there is a unique pinned fluxon for each length, it is due to the fact that the pinned fluxons are buiklt from periodic orbits (that may be travelled in various waus before leaving the inhomogeneity). For $\gamma>0$, there is minimum length $L_{\min }$ such that there are at least two pinned fluxons for each length $L>L_{\min }$ (one for $L$ minimal). The $L-h$ curves for $d=4$ and various $\gamma$ values are given in Figure 22. Only lengths of the pinned fluxons that use less than a full periodic orbit are plotted.

In the following theorem, we summarise the existence of pinned fluxons for $d>1$ and give their stability.

Theorem 11 Let $\widehat{d}$ be the solution of $-\frac{5 \pi}{2}+\arcsin \frac{1}{d}+\sqrt{d^{2}-1}+d-1=0(\widehat{d} \approx 4.37)$ and for $d>1$, let $\gamma_{\max }(d)$ be the (implicit) solution of $-2 \pi \gamma-\gamma\left(\arcsin \gamma-\arcsin \frac{\gamma}{d}\right)+\sqrt{d^{2}-\gamma^{2}}-\sqrt{1-\gamma^{2}}+(d-1)=0$.

- For $d>1$ and $\gamma=0$, there is at least one pinned fluxon for each $L \geq 0$ and all pinned fluxons are unstable;

- For $1<d \leq \widehat{d}$ and $0<\gamma<\gamma_{\max }(d)$, there is a minimal length $L_{\min }(\gamma)>0$ such that for all 

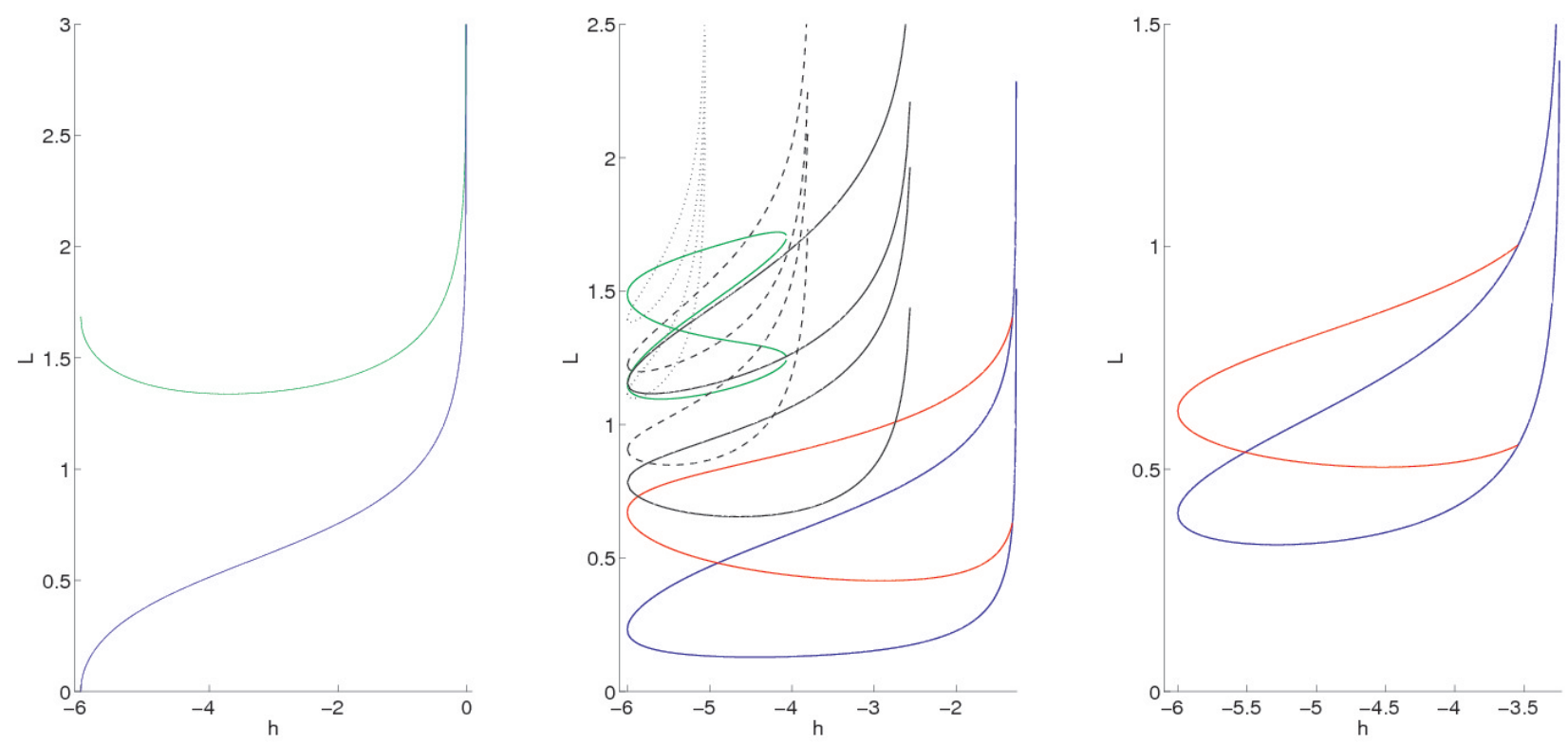

Figure 22: $L$ - $h$ curves at $d=4$ and $\gamma=0$ (left), $\gamma=0.2$ (middle) and $\gamma=0.5$ (right). The blue and red curves are associated with pinned fluxons with $\arcsin \gamma / d<\phi_{\text {in }}<\pi$. The red curve are pinned fluxons with $p_{\text {in }}<0$ and $\arcsin \gamma<\phi_{\text {in }}<\pi$. The pinned fluxons in the blue curve have $p_{\text {in }}>0$ for $\phi_{\text {in }}>\arcsin \gamma$ and $p_{\text {in }}<0$ for $\phi_{\text {in }}<\arcsin \gamma$. The green curves are associated with pinned fluxons with $\phi_{\text {in }}>\pi$. In the middle panel $(\gamma=0.2)$ there are also black curves, which are associated with pinned fluxons with $\phi_{\text {in }}<\arcsin \frac{\gamma}{d}$. The solid black curves are lengths for pinned fluxons with $-2 \pi+\arcsin \frac{\gamma}{d}<$ $\phi_{\text {in }}<\arcsin \frac{\gamma}{d}$, the dashed ones for pinned fluxons with $-4 \pi+\arcsin \frac{\gamma}{d}<\phi_{\text {in }}<-2 \pi+\arcsin \frac{\gamma}{d}$, the dotted ones for pinned fluxons $-6 \pi+\arcsin \frac{\gamma}{d}<\phi_{\text {in }}<-4 \pi+\arcsin \frac{\gamma}{d}$.

$L>L_{\min }$ there exist at least two pinned fluxons (one for $L=L_{\min }$ ). For each $L \geq L_{\min }$, there is at least one stable pinned fluxon.

- For $d>\widehat{d}$ and $0<\gamma \leq 1$, there is a minimal length $L_{\min }(\gamma)>0$ such that for all $L>L_{\min }$ there exist at least two pinned fluxons (one for $L=L_{\min }$ ). For each $L \geq L_{\min }$, there is at least one stable pinned fluxon.

In Figure 22, the stable pinned fluxons are the pinned fluxons on the increasing part of the lower right blue curve. Note that these pinned fluxons are non-monotonic past the meeting point with the red curve, hence for most lengths. The fluxons on the other blue curve and red and green curves are unstable. As before, the proof of the stability properties of Theorem 11 is based on Theorem 3.1 from [19]. The proof of Theorem 11 is very similar to the proof of Theorems 8 and 9. The main difference is that we can not track our stability arguments back to the case $d=0$ (i.e. Lemma 4 ) as we did before. The role of Lemma 4 will now be taken over by Lemma 12 in Appendix A, in which it is explicitly established tht the pinned fluxon on the blue curve has exactly one positive eigenvalue for $\gamma=0$ and $d$ near one.

The stability of the fluxons on the black curves can not easily be related to fluxons at $\gamma=0$ (they "split" in a homoclinic "dip" and a fluxon for $\gamma=0$ ). So a stability analysis for this case goes outside the scope of this paper. In section 5, we will show numerically that there are some stable fluxons on the black curve. 


\subsection{A microresonator approximating a localised inhomogeneity}

There have been quite a number of investigations on the influence of a localised inhomogeneity, i.e., $D(x)=(1+\mu \delta(x))$ or $D(x)=\left(1+\sum_{i=1}^{N} \mu_{i} \delta\left(x-x_{i}\right)\right)$ in (1). In this section we will confirm that our existence and stability results, applied to short microresonators with large $d$, reproduce in the limit for $L \rightarrow 0$ and $d \rightarrow \infty$ the existence and stability results for pinning by microshorts in [22]. In [22] it is shown that for $D(x)=(1+\mu \delta(x))$ and $\gamma, \mu$, and $\alpha$ of order $\varepsilon$, with $\varepsilon$ small and $\frac{\pi \gamma}{\mu} \leq \frac{4}{3 \sqrt{3}}+\mathcal{O}(\varepsilon)$, there are one stable and one unstable pinned fluxon, both approximated by $\phi_{0}\left(x-X_{0}\right)+\mathcal{O}(\varepsilon)$, where $X_{0}$ are the two solutions of $-\frac{\pi \gamma}{2 \mu}+\operatorname{sech}^{2} X \tanh X$.

To approximate the localised inhomogeneities of $\delta$-function type with finite length ones, we look at microresonators with length $L=1 /(2 \mathfrak{d})$ and $d=1+\mu \mathfrak{d}$ for $\mathfrak{d}$ large. Thus the microresonators have short lengths and we can restrict to pinned fluxons with

$$
\phi_{\text {in }}=\arccos \left(\frac{2 \pi \gamma+d-1+h}{d-1}\right)=\arccos \left(1+\frac{2 \pi \gamma+h}{\mu \mathfrak{d}}\right), \quad p_{\text {in }}>0,
$$

and

$$
\phi_{\text {out }}=\arccos \left(\frac{d-1+h}{d-1}\right)=\arccos \left(1+\frac{h}{\mu \mathfrak{d}}\right) .
$$

Hence the pinned fluxons of [22] correspond to solutions on the lower blue curve in Figure 22. Introducing $h=\mu \mathfrak{d} \mathfrak{h}$, we get that $-2<\mathfrak{h}<0$ and we are interested in $\mathfrak{h}$ away from 0 as $\mathfrak{h}$-values close to zero correspond to long lengths. Using the expressions for $\phi_{\text {in }}, p_{\text {in }} \phi_{\text {out }}$, and $p_{\text {out }}$ and the ODE for the pinned fluxon, we can derive an asymptotic expression for the length $L(\mathfrak{h})$ if $\mathfrak{d}$ is large and $\gamma, \mu$ are order $\varepsilon$, where $\varepsilon$ is small:

$$
L(\mathfrak{h})=\frac{\pi \gamma}{-\mathfrak{h} \mu \mathfrak{d} \sqrt{2(2+\mathfrak{h})}}+\mathcal{O}\left(\mathfrak{d}^{-2}+\varepsilon \mathfrak{d}^{-1}\right), \quad \varepsilon, \mathfrak{d}^{-1} \rightarrow 0 .
$$

Thus $L(\mathfrak{h})$ has a minimum at $\mathfrak{h}=-\frac{4}{3}+\mathcal{O}\left(\mathfrak{d}^{-1}+\varepsilon\right)$ and the condition $L(\mathfrak{h})=1 / 2 \mathfrak{d}$ can be satisfied if the cubic $\mathfrak{h}^{2}(2+\mathfrak{h})=2 \frac{\pi^{2} \gamma^{2}}{\mu^{2}}+\mathcal{O}\left(\mathfrak{d}^{-1}+\varepsilon\right)$ can be solved for some $\mathfrak{h}<0$. For $\mathfrak{h}<0$, this cubic has a maximum at $\mathfrak{h}=-\frac{4}{3}+\mathcal{O}\left(\mathfrak{d}^{-1}+\varepsilon\right)$, thus $L(\mathfrak{h})=\frac{1}{2 \mathfrak{d}}$ has two solutions with $\mathfrak{h}$ between -2 and 0 iff $\frac{\pi \gamma}{\mu} \leq \frac{4}{3 \sqrt{3}}+\mathcal{O}\left(\mathfrak{d}^{-1}+\varepsilon\right)$ (i.e., there are no solutions for $\gamma / \mu$ too large). From the analysis in the previous section, we can conclude that this corresponds to one stable pinned fluxon (least negative value of $\mathfrak{h}$ ) and one unstable pinned fluxon.

Finally, for $\gamma=\mathcal{O}(\varepsilon)$, with $\varepsilon$ small, both the unstable manifold to $\arcsin \gamma$ and the stable manifold to $2 \pi+\arcsin \gamma$ are close to the heteroclinic connection for the unperturbed sine-Gordon equation. Thus for $x>L$, we have $\phi_{\text {pin }}(x)=\phi_{0}\left(x-X_{0}\right)+\mathcal{O}(\varepsilon)$, where $\phi_{0}$ is the shape of the stationary fluxon in the sine-Gordon equation (and a similar relation for $x<-L$ ). Substituting this into the equation for $\phi_{\text {out }}$, with $L=1 / 2 \mathfrak{d}$ (hence $\mathfrak{h}$ is a solution of the cubic introduced earlier), we get that $X_{0}$ is one of the two solutions of $-\frac{\pi \gamma}{2 \mu}+\operatorname{sech}^{2} X \tanh X=0$.

\section{Conclusions and further work}

This paper exhibits a full analysis for the existence and stability of pinned fluxons in microresistors and microresonators for which the Josephson tunneling critical current is modelled by a step-function. It is shown that for fixed $d$ (Josephson tunneling critical current inside the inhomogeneity) and fixed bias current $\gamma>0$, there is an interval of lengths for which a rich family of pinned fluxons exists. In the case when an induced current is present, there is a lower bound on the length of inhomogeneities for which pinned fluxons can exist. If the inhomogeneity is too short, no pinned fluxons can be sustained. The lower bound on the length increases if the induced current increases. For microresistors with a sufficiently large induced current, there is also an upper bound on the length for pinned fluxons and 
the upper and lower bounds collide when the maximal value of the induced current for which pinned fluxons can exist, is attained

Compared to the case of homogeneous wave equations, a new phenomenon is observed: longer microresistors and microresonators have non-monotonic stable pinned fluxons. In the case of microresistors $(d<1)$, the non-monotonic stable pinned fluxons have a "bump" inside and behind the inhomogeneity and the values in the bump exceed the asymptotic state $2 \pi+\arcsin \gamma$. In the case of the microresonators $(d>1)$, the stable pinned fluxons have a "dip" before and near the inhomogeneity and the values in the dip are between $\arcsin \gamma / d$ and $\arcsin \gamma$, i.e., below the left asymptotic state.

To complement and illustrate the analytical results in the previous sections, we have numerically solved the stationary equation (4) for the pinned fluxons and the corresponding linear eigenvalue problem (6) using a simple finite difference method and presented the results in Figures 23-26. Without loss of generality as far as stability is concerned, we depict the eigenvalues for $\alpha=0$, i.e., $\Lambda=\lambda^{2}$. Thus an instability is indicated by the presence of a pair of eigenvalues with non-zero real parts.

First, we consider the case of inhomogeneous Josephson junctions for a microresistor with $d=0$. As is shown in Figure 17, when $\gamma=0.15$ and the defect length parameter $L=4.2$, there are four possible pinned fluxons. In Figure 23, the numerically obtained profiles of pinned fluxons are a shown; all of them are clearly non-monotonous. The insets show the eigenvalues of the fluxons in the complex plane. Only one of them has no eigenvalues with non-zero real parts, confirming that there is exactly one stable pinned fluxon, which is non-monotonous for these parameter values. The four pinned fluxons belong to two different families, the ones with the smallest bump, i.e. panel (a), are on the blue curve and the others, i.e. panel (b), on the green curve in Figure 17.

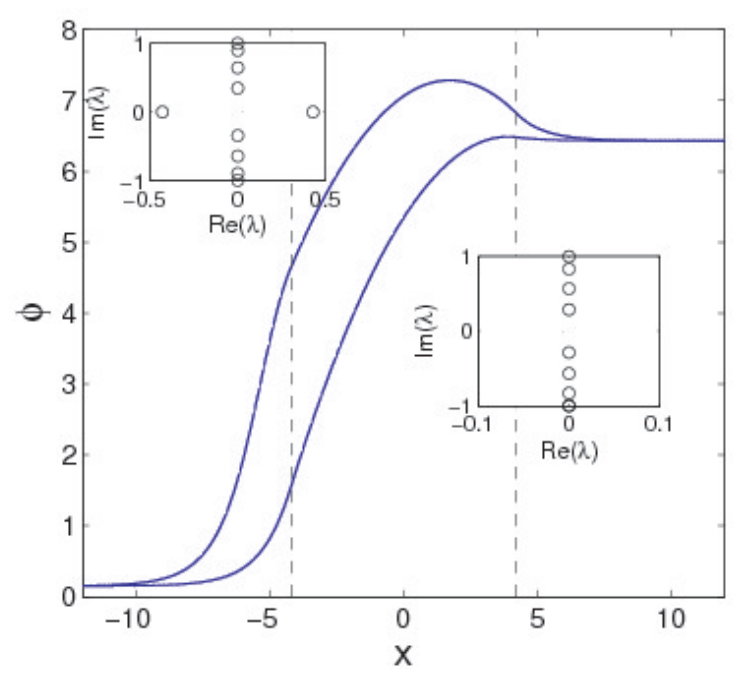

(a)

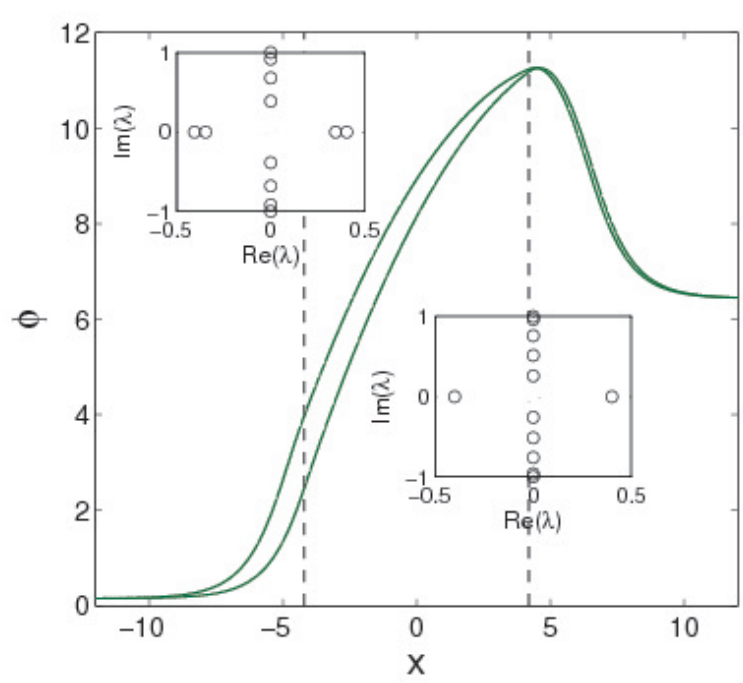

(b)

Figure 23: The four pinned fluxons admitted by the Josephson system with $d=0 L=4.2$, and $\gamma=0.15$. The insets show the eigenvalues of each fluxon; the top inset is related to the upper fluxon and the bottom inset to the lower fluxon. The vertical dashed lines show the edges of the defect.

In Figure 17, the existence and the stability of the pinned fluxons for fixed $d$ and $\gamma$ are presented in the $(h, L)$-plane and it is shown that each pair of the fluxons collide in a saddle-node bifurcation at a critical $L$ for a fixed $\gamma$. To complement these results, we take $L=4.2$ and numerically follow the largest eigenvalue $\Lambda=\lambda^{2}$ of the various fluxons when the induced current $\gamma$ changes. The results are shown in Figure 24. As before, the colouring corresponds to the colouring in Figure 17. Figure 24 shows that there is a critical current for the existence of a pinned fluxon for a given length and depth of the inhomogeneity. The blue and green fluxons disappear in a saddle-node bifurcation. This happens 
at a smaller value of $\gamma$ for the green fluxons (solutions in panel (b) in Figure 23) than for the blue fluxons (panel (a)). A physical interpretation of the saddle-node bifurcation is that the inhomogeneity is too short or long to pin a fluxon when the applied current exceeds the critical value. For $\gamma=0.15$, there are no red fluxons at this length, but they will exist for smaller values of $\gamma$. The red fluxons disappear when the fluxon "splits" in a homoclinic connection to $2 \pi+\arcsin \gamma$ and a blue pinned fluxon. Only one curve of red fluxons is visible. In theory, there is a second curve, but this exist in a tiny $\gamma$-interval only and hence is not visible.

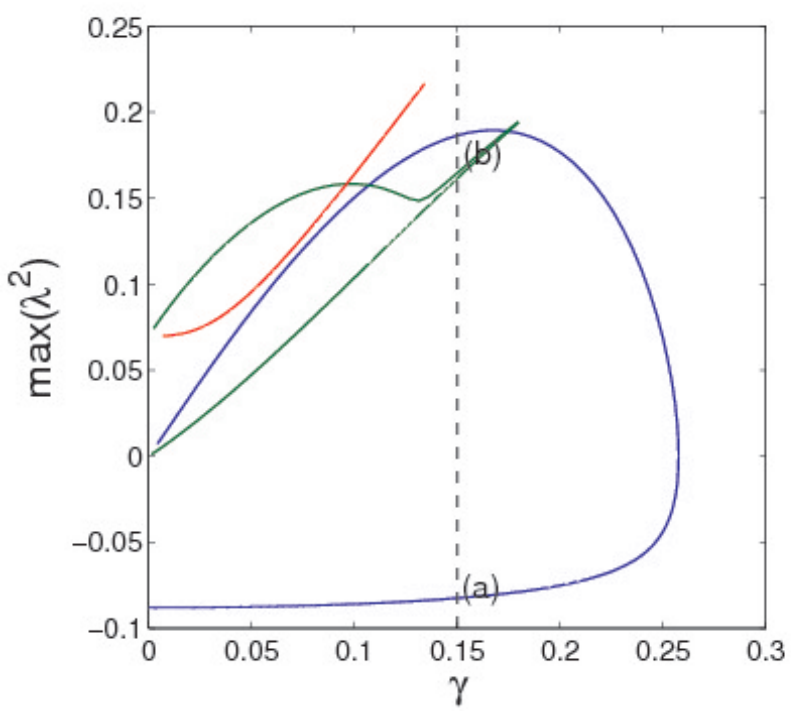

Figure 24: The largest eigenvalue $\Lambda=\lambda^{2}$ of the various fluxons as function of the induced current $\gamma$. The maximal eigenvalue at $\gamma=0.15$ of the fluxons in Figure 23 is at the intersection between the curves and the vertical dashed line. Note that the blue and green fluxons disappear in a saddle-node bifurcation.

In Figures 25-26, we consider the case of a microresonator with $d=4$. From the middle panel in Figure 22, it follows that there exist five pinned fluxons when $\gamma=0.2$, and $L=0.75$. In Figure 25 we show the numerically computed profiles of those pinned fluxons and their eigenvalues, where the colouring is as in Figure 22. The blue non-monotonic fluxon is stable while the blue monotonic one and red one are unstable. This confirms our analytical findings (see Theorem 11: there is at least one stable pinned fluxon). Moreover, it shows that there can be more than one stable fluxon: one of the fluxons on the black curve is stable too. So for $d>1$, there is bi-stability for some values of $L$ and $\gamma$.

In Figure 26 we also present the critical eigenvalues of the five fluxons as a function of $\gamma$ when $L=0.75$ is fixed. Similarly as in Figure 24, the pairs of blue and black fluxons collide in a saddle-node bifurcation, while the red fluxon breaks up at the maximal value of $\gamma$.

For future research, it is of interest to expand our study to the case of two-dimensional Josephson junction with inhomogeneities. A particular example is the so-called window Josephson junction, which is a rectangular junction surrounded by an inhomogeneous 'idle' region with $d=0$. The interested reader is referred to $[4,5,8]$ and references therein for reviews of theoretical and experimental results on window Josephson junctions. Recently, fluxon scatterings in a 2D setup in the presence of a non-zero defect has been considered as well in [24].

One can also apply our method to study the existence of trapped solitons by inhomogeneities in Schrödinger equations, such as pinned optical solitons in a nonlinear Bragg media with a finite-size inhomogeneity (see, e.g., [13] and references therein) and trapped Bose-Einstein condensates by a finite square-well potential (see, e.g., $[9,23]$ ). In general, the ideas presented in this paper are applicable 


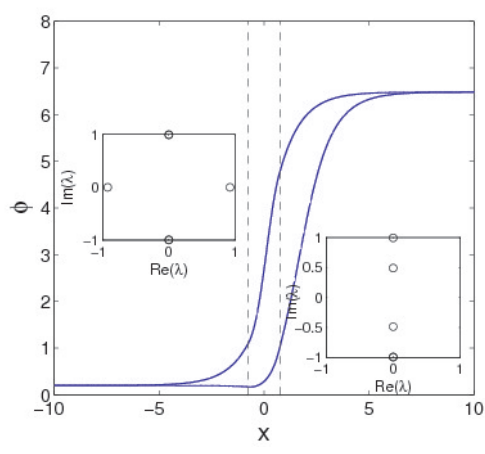

(a)

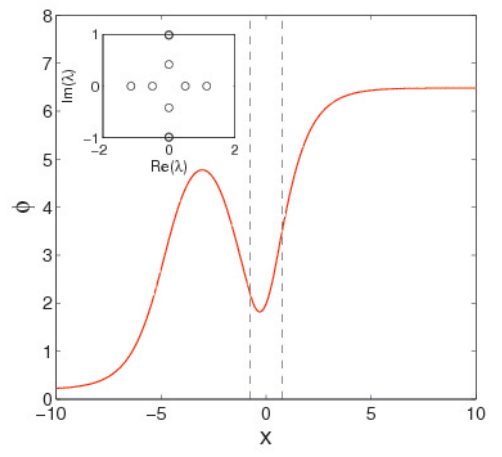

(b)

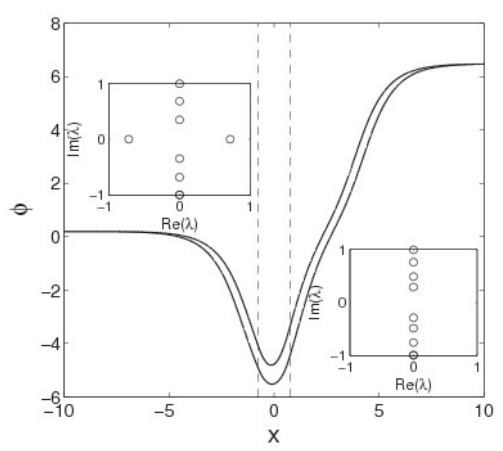

(c)

Figure 25: The same as in Figure 23, but for a microresonator with $d=4, L=0.75$, and $\gamma=0.2$, where there are five pinned fluxons.

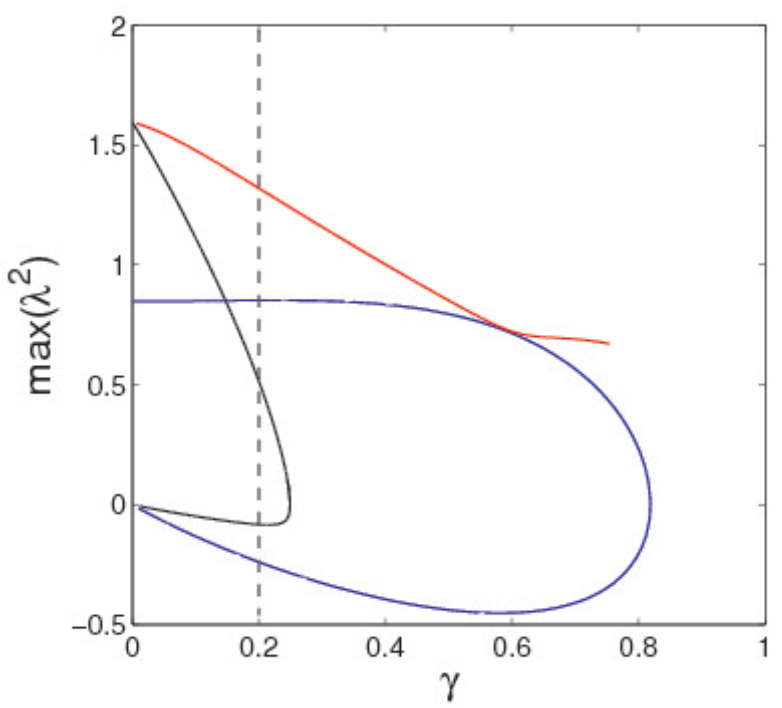

Figure 26: The same as in Figure 24, but for the five pinned fluxons in Figure 25.

to any system with locally (piecewise constant) varying parameters in the equations as can be seen in preprints by some of us [21] and [14].

Finally, the simulations in section 2 show how inhomogeneities can capture travelling fluxons. This suggests that the pinned fluxons analysed in this paper can be attractive or repelling, just as observed in [22] in case of the localised inhomogeneities. We are currently investigating the attractive and repelling interaction of the travelling fluxons with the pinned fluxons and will report on this in a future paper.

Acknowledgement We would like to thank Daniele Avitabile for the use of his suite of simulation codes.

\section{A Largest eigenvalue of linearisation with no induced current}

Proof of Lemma 4 Let $\gamma=0, d=0$, and fix the length $L$ of the inhomogeneity. We denote the unique pinned fluxon with length $L$ by $\phi_{\text {pin }}(x)$ (suppressing all other parameters). From (12), we see that $\phi_{\text {pin }}$ equals the sine-Gordon fluxon outside the inhomogeneity $(|x|>L)$ and the linearisation about the sine-Gordon fluxon is well-studied. The shifted pinned fluxon $\phi_{\operatorname{pin}}(x)-\pi$ is an odd function, hence 
a quick inspection shows that the operator $\mathcal{L}_{\text {pin }}(x)$ is even in $x$ (we suppress all other parameters in $\left.\mathcal{L}_{\text {pin }}\right)$. All eigenvalues of $\mathcal{L}_{\text {pin }}$ are simple, thus $\mathcal{L}_{\text {pin }}(x)$ being even implies that all eigenfunctions are odd or even. The eigenfunction for the largest eigenvalue does not have any zeroes, thus this eigenfunction is even.

For fixed $\Lambda$, the linear ODE associated with $\left(\mathcal{L}_{\text {pin }}-\Lambda\right) \Psi=0$ has two linearly independent solutions. The asymptotic limits of $\phi_{\text {pin }}$ for $x \rightarrow \pm \infty$ correspond to saddle points in the ODE (4) and the decay rate to these fixed points is like $e^{-x}$. This implies that for $\Lambda>-1$, there is one solution of the ODE $\left(\mathcal{L}_{\text {pin }}-\Lambda\right) \Psi=0$ that is exponentially decaying at $+\infty$ and there is one solution that is exponentially decaying at $-\infty$. We denote the exponentially decaying function at $-\infty$ by $v_{-}(x ; L, \Lambda)$.

In [20], the linearisation of the sine-Gordon equation about the the fluxon $\phi_{0}$ is studied in great detail. Using the results in this paper, we can derive an explicit expression for the solutions $v_{-}(x ; L, \Lambda)$ (see also [10]), they are

$$
\begin{aligned}
& v_{-}(x ; L, 0)=\operatorname{sech}\left(x+x^{*}(L)\right), \quad x<-L \\
& v_{-}(x ; L, \Lambda)=e^{\mu\left(x+x^{*}(L)\right)}\left[\tanh \left(x+x^{*}(L)\right)-\mu\right], \quad x<-L \quad \text { where } \quad \mu=\sqrt{\Lambda+1},
\end{aligned}
$$

where $x^{*}(L)$ is given in Lemma 1. In the inhomogeneity, the linearised operator is simply $\mathcal{L}_{\text {pin }}=D_{x x}$, hence the even solutions of $\mathcal{L}_{\text {pin }}-\Lambda$ are

$$
\begin{aligned}
v_{\text {inhom }}(x ; \Lambda) & =A \cos (\sqrt{-\Lambda} x), \quad|x|<L, \quad \text { if } \Lambda<0 ; \\
v_{\text {inhom }}(x ; 0) & =A, \quad|x|<L ; \\
v_{\text {inhom }}(x ; \Lambda) & =A \cosh (\sqrt{\Lambda} x), \quad|x|<L, \quad \text { if } \Lambda>0 .
\end{aligned}
$$

To have a continuously differentiable solution of $\left(\mathcal{L}_{\text {pin }}-\Lambda\right) \psi=0$ in $H^{2}(\mathbb{R})$, we have to match $v_{-}$and $v_{\text {inhom }}$ and its derivatives at $x=-L$ (the conditions for $x=L$ following immediately from this as the eigenfunction is even). This gives:

- If $\Lambda=0$ (thus $\mu=1)$ :

$$
A=\operatorname{sech} \xi^{*} \text { and } 0=-\operatorname{sech} \xi^{*} \tanh \xi^{*}
$$

with $\xi^{*}=-L+x^{*}(L)$. This implies that $\xi^{*}=0$ and $A=0$. From the relation for $x^{*}(L)$ in Lemma 1 , it follows $\xi^{*} \neq 0$ only if $L=0$, hence when there is no inhomogeneity. This confirms that the stationary sine-Gordon fluxon (the pinned fluxon for $L=0$ ) has an eigenvalue zero, but none of the pinned fluxons with $L>0$ will have an eigenvalue zero for its linearisation $\mathcal{L}_{\text {pin }}$.

- If $\Lambda>0$ (thus $\mu>1$ ), with $y^{*}=L \sqrt{\mu^{2}-1}$ and again $\xi^{*}=-L+x^{*}(L)$ :

$$
\begin{aligned}
A \cosh y^{*} & =e^{\mu \xi^{*}}\left[\tanh \xi^{*}-\mu\right] \\
-\sqrt{1-\mu^{2}} A \sinh y^{*} & =e^{\mu \xi^{*}}\left[\mu\left(\tanh \xi^{*}-\mu\right)+\operatorname{sech}^{2} \xi^{*}\right]
\end{aligned}
$$

Hence $\mu$ (thus $\Lambda$ ) is determined by

$$
\mu\left[\tanh \xi^{*}-\mu\right]+\operatorname{sech}^{2} \xi^{*}=-\sqrt{\mu^{2}-1}\left[\tanh \xi^{*}-\mu\right] \tanh y^{*} .
$$

Using Lemma 1, this can be written as a relation between $\mu$ and $\phi_{\text {in }}$ (and hence $\mu$ and $L$ as there is a bijection between $\phi_{\text {in }} \in(0, \pi)$ and $\left.L>0\right)$ :

$$
\begin{aligned}
&-\mu\left[\mu+\frac{1}{2}\right.\left.\sqrt{2\left(1+\cos \phi_{\text {in }}\right)}\right]+\frac{1}{2}\left(1-\cos \phi_{\text {in }}\right)= \\
& \sqrt{\mu^{2}-1}\left[\mu+\frac{1}{2} \sqrt{2\left(1+\cos \phi_{\text {in }}\right)}\right] \tanh \left(\sqrt{\mu^{2}-1} \frac{\pi-\phi_{\text {in }}}{\sqrt{2\left(1-\cos \phi_{\mathrm{in}}\right)}}\right) .
\end{aligned}
$$


It can be seen immediately that the right-hand side of (22) is positive. The left-hand side of (22) is always negative for $\mu>1$ as

$$
-\mu(\mu+T)+1-T^{2}=1-\mu^{2}-\mu T-T^{2} \leq-T-T^{2}<0,
$$

where we wrote $T=\frac{1}{2} \sqrt{2\left(1+\cos \phi_{\text {in }}\right)}$, hence $\frac{1}{2}\left(1-\cos \phi_{\text {in }}\right)=1-T^{2}$. Thus (22) has no solutions and there do no exist any eigenvalues $\Lambda>0$.

- If $-1<\Lambda<0$ (thus $0<\mu<1$ ), again with $\xi^{*}=-L+x^{*}(L)$ and now $y^{*}=L \sqrt{1-\mu^{2}}$ :

$$
A \cos y^{*}=e^{\mu \xi^{*}}\left[\tanh \xi^{*}-\mu\right] \quad \text { and } \quad \sqrt{1-\mu^{2}} A \sin y^{*}=e^{\mu \xi^{*}}\left[\mu\left(\tanh \xi^{*}-\mu\right)+\operatorname{sech}^{2} \xi^{*}\right] .
$$

Hence $\mu$ (thus also $\Lambda$ ) is determined by

$$
\mu\left[\tanh \xi^{*}-\mu\right]+\operatorname{sech}^{2} \xi^{*}=\sqrt{1-\mu^{2}}\left[\tanh \xi^{*}-\mu\right] \tan y^{*} .
$$

Using the same relations as before, this can be written as a relation between $\mu$ and $\phi_{\text {in }}$ :

$$
\begin{aligned}
-\mu\left[\mu+\frac{1}{2}\right. & \left.\sqrt{2\left(1+\cos \phi_{\text {in }}\right)}\right]+\frac{1}{2}\left(1-\cos \phi_{\text {in }}\right)= \\
& -\sqrt{1-\mu^{2}}\left[\mu+\frac{1}{2} \sqrt{2\left(1+\cos \phi_{\text {in }}\right)}\right] \tan \left(\sqrt{1-\mu^{2}} \frac{\pi-\phi_{\text {in }}}{\sqrt{2\left(1-\cos \phi_{\text {in }}\right)}}\right) .
\end{aligned}
$$

Bringing all terms to the left and writing $T(L)=\frac{1}{2} \sqrt{2\left(1+\cos \phi_{\text {in }}(L)\right)} \in(0,1)$ gives on the left

$$
F(L, \mu):=-\mu[\mu+T]+1-T^{2}+\sqrt{1-\mu^{2}}[\mu+T] \tan \left(\sqrt{1-\mu^{2}} L\right) .
$$

Taking $\mu=1$ in this expression gives $F(L, 1)=-T-T^{2} \leq 0$. If $L<\frac{\pi}{2}$, then $F(L, 0)=1-$ $T^{2}+T \tan L>0$ as $T \in(0,1)$. If $L \geq \frac{\pi}{2}$, then $\phi_{\text {in }}<\frac{\pi}{2}$ and $T>\frac{1}{2} \sqrt{2}$, thus $F\left(L, \frac{\sqrt{L^{2}-(\pi / 2-\varepsilon)^{2}}}{L}\right) \geq$ $-2+\frac{(\pi / 2-\varepsilon) \sqrt{2}}{2 L} \tan \left(\frac{\pi}{2}-\varepsilon\right)=\mathcal{O}\left(\frac{1}{L \varepsilon}\right)$, for $\varepsilon \rightarrow 0$. As $L$ is fixed, we can choose $\varepsilon$ such that this expression is positive. Thus we can conclude that for all $L>0$, there is at least one $\mu \in(0,1)$ that solves $F(L, \mu)=0$. If $L$ gets very large, then there will be many solutions, but we are interested in the largest one.

Lemma 12 For $\gamma=0$ and $d=1+\varepsilon$ with $\varepsilon$ small, the linearisation $\mathcal{L}_{\text {pin }}(x ; L, 0,1+\varepsilon)$ about the monotone pinned fluxon $\phi_{\operatorname{pin}}(x ; L, 0,1+\varepsilon)$ has a largest eigenvalue of the form $\varepsilon \Lambda_{1}+\mathcal{O}\left(\varepsilon^{2}\right)$ with

$$
\Lambda_{1}=\frac{\operatorname{sech}^{2} L\left[-L^{2} \operatorname{sech}^{4} L\left(1+\tanh ^{2} L\right)+2 L \tanh L\left(\operatorname{sech}^{4} L+2\left(1+\operatorname{sech}^{2} L\right)\right)+\tanh ^{2} L\left(6+\operatorname{sech}^{2} L\right)\right]}{16\left(L \operatorname{sech}^{2} L+\tanh L\right)} .
$$

See Figure 27 for a sketch of $\Lambda_{1}$. Furthermore, if there are any other eigenvalues, then they must be near -1 . Thus for $\gamma=0$ and $d$ close to 1 , the monotone pinned fluxons with $d>1$ are linearly unstable. The nonlinear stability of Theorem 9 is confirmed by the sign of $\Lambda_{1}$ for $d<1$.

Proof The monotone pinned fluxon for $\gamma=0$ and $d=1+\varepsilon$ with $\varepsilon \ll 1$ can be written as

$$
\phi_{\text {pin }}(x ; L, 0,1+\varepsilon)=\left\{\begin{array}{cl}
\phi_{0}\left(x+\varepsilon x_{1}^{*}(L, \varepsilon)\right), & x<-L, \\
\phi_{0}(x)+\varepsilon \phi_{1}(x ; L, \varepsilon), & |x|<L, \\
\phi_{0}\left(x-\varepsilon x_{1}^{*}(L, \varepsilon)\right), & x>L .
\end{array}\right.
$$




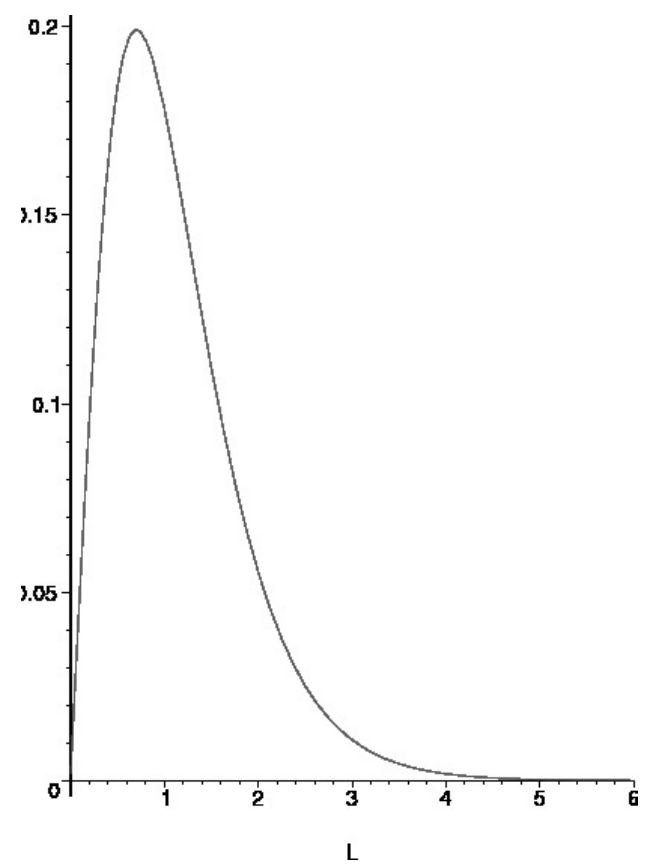

Figure 27: The eigenvalue factor $\Lambda_{1}$ as function of $L$.

Here $\phi_{1}(x ; L, \varepsilon)$ is an odd function satisfying

$$
\varepsilon D_{x x} \phi_{1}-(1+\varepsilon) \sin \left(\phi_{0}+\varepsilon \phi_{1}\right)+\sin \phi_{0}=0,|x|<L,
$$

and $x_{1}^{*}(L, \varepsilon)$ is such that $\phi_{0}\left(-L+\varepsilon x_{1}^{*}\right)=\phi_{\text {in }}=\phi_{0}(-L)+\varepsilon \phi_{1}(-L)$. To find an approximation for $\phi_{1}$ and $x_{1}^{*}$, we introduce the notation $\phi_{0}^{*}=\phi_{0}(-L)$, and $\phi_{1}^{*}=\phi_{1}(-L)$, thus $\varepsilon \phi_{1}^{*}=\phi_{\text {in }}-\phi_{0}^{*}$. The half length $L$ is

$$
L=\int_{\phi_{\mathrm{in}}}^{\pi} \frac{d \phi}{\sqrt{2(h+(1+\varepsilon)(1-\cos \phi)}}=\int_{\phi_{\mathrm{in}}}^{\pi} \frac{d \phi}{\sqrt{2\left(1-\cos \phi_{\mathrm{in}}+(1+\varepsilon)\left(\cos \phi_{\mathrm{in}}-\cos \phi\right)\right)}},
$$

where we used that $h=\varepsilon\left(\cos \phi_{\text {in }}-1\right)$. With $\phi_{\text {in }}=\phi_{0}^{*}+\varepsilon \phi_{1}^{*}$, this becomes

$$
\begin{aligned}
L & =\int_{\phi_{0}^{*}}^{\pi} \frac{d \phi}{\sqrt{2(1-\cos \phi)}}-\frac{\varepsilon}{2} \int_{\phi_{0}^{*}}^{\pi} \frac{\cos \phi_{0}^{*}-\cos \phi}{(2(1-\cos \phi))^{3 / 2}} d \phi-\int_{\phi_{0}^{*}}^{\phi_{0}^{*}+\varepsilon \phi_{1}^{*}} \frac{d \phi}{\sqrt{2(1-\cos \phi)}}+\mathcal{O}\left(\varepsilon^{2}\right) \\
& =L-\frac{\varepsilon}{2} \int_{\phi_{0}^{*}}^{\pi} \frac{\cos \phi_{0}^{*}-\cos \phi}{(2(1-\cos \phi))^{3 / 2}} d \phi-\varepsilon \int_{0}^{\phi_{1}^{*}} \frac{d \phi}{\sqrt{2\left(1-\cos \phi_{0}^{*}\right)}}+\mathcal{O}\left(\varepsilon^{2}\right) .
\end{aligned}
$$

Rearranging this expression and using that $\cos \phi_{0}^{*}=1-2 \operatorname{sech}^{2}(L)$, we get an approximation for $\phi_{1}^{*}$

$$
\phi_{1}^{*}=-\frac{\operatorname{sech} L}{8}\left[2 L\left(1+\tanh ^{2} L\right)-2 \tanh L\right]+\mathcal{O}(\varepsilon) .
$$

Furthermore, $x_{1}^{*}$ is given by $\phi_{0}\left(-L+\varepsilon x_{1}^{*}\right)=\phi_{0}^{*}+\varepsilon \phi_{1}^{*}$. An expansion of $\phi_{0}\left(-L+\varepsilon x_{1}^{*}\right)$ shows that

$$
\phi_{0}^{*}+\varepsilon x_{1}^{*} \phi_{0}^{\prime}(-L)=\phi_{0}^{*}-\frac{\varepsilon \operatorname{sech} L}{8}\left[2 L\left(1+\tanh ^{2} L\right)-2 \tanh L\right]+\mathcal{O}\left(\varepsilon^{2}\right) .
$$

With $\phi_{0}^{\prime}(-L)=2 \operatorname{sech}(L)$, this shows that

$$
x_{1}^{*}=-\frac{1}{16}\left[2 L\left(1+\tanh ^{2} L\right)-2 \tanh L\right]+\mathcal{O}(\varepsilon) .
$$

Next, we derive an approximation for the function $\phi_{1}$, using the differential equation (23). Expanding (23) in $\varepsilon$ gives

$$
D_{x x} \phi_{1}-\phi_{1} \cos \phi_{0}-\sin \phi_{0}=\mathcal{O}(\varepsilon) \quad \text { or } \quad \mathcal{L}_{0} \phi_{1}=\sin \phi_{0}+\mathcal{O}(\varepsilon)
$$


with $\mathcal{L}_{0}=D_{x x}-\cos \phi_{0}$. The homogeneous problem $\mathcal{L}_{0} \psi=0$ has two independent solutions: $\psi_{b}(x)=$ $\operatorname{sech} x$ and $\psi_{u}(x)=x \operatorname{sech} x+\sinh x$. In this, $\psi_{b}(x)=\frac{1}{2} \frac{d}{d x} \phi_{0}(x)$ is bounded and $\psi_{u}(x)$ unbounded as $x \rightarrow \pm \infty$. By the variation-of-constants method, we find the general solution to (24),

$$
\phi_{1}(x)=x \operatorname{sech} x+A \operatorname{sech} x+B[x \operatorname{sech} x+\sinh x]+\mathcal{O}(\varepsilon),
$$

with $A, B \in \mathbb{R}$. As $\phi_{1}$ must be odd, it follows that $A=0$. Furthermore, the boundary condition at $x=-L$ gives $\phi_{1}^{*}=-B(L \operatorname{sech} L+\sinh L)-L \operatorname{sech} L+\mathcal{O}(\varepsilon)$, hence

$$
B=\frac{\operatorname{sech} L\left(L \tanh ^{2} L-\tanh L-3 L\right)}{4(L \operatorname{sech} L+\sinh L)} .
$$

Altogether we can conclude that $\phi_{1}(x)=\phi_{11}(x)+\mathcal{O}(\varepsilon)$ with

$$
\phi_{11}(x)=x \operatorname{sech} x+\frac{\operatorname{sech} L\left(L \tanh ^{2} L-\tanh L-3 L\right)}{4(L \operatorname{sech} L+\sinh L)}[x \operatorname{sech} x+\sinh x] .
$$

To find the largest eigenvalue of $\mathcal{L}_{\text {pin }}(x ; h, 0,1+\varepsilon)$, we will use perturbation theory. First we observe that for any $L \geq 0$, the linearisation $\mathcal{L}_{0}:=\mathcal{L}_{\text {pin }}(x ; L, 0,1)$ about the fluxon $\phi_{0}$ has largest eigenvalue $\Lambda=0$ with eigenfunction is $\phi_{0}^{\prime}$. We have for $|x|<L$

$$
\mathcal{L}_{\text {pin }}(x ; h, 0,1+\varepsilon)=D_{x x}-(1+\varepsilon) \cos \left(\phi_{0}+\varepsilon \phi_{1}\right)=\mathcal{L}_{0}(x)-\varepsilon\left(\cos \phi_{0}-\phi_{1} \sin \phi_{0}\right)+\mathcal{O}\left(\varepsilon^{2}\right)
$$

and for $x<-L$

$$
\mathcal{L}_{\text {pin }}(x ; h, 0,1+\varepsilon)=\mathcal{L}_{0}\left(x+\varepsilon x_{1}^{*}\right)=\mathcal{L}_{0}(x)+\varepsilon x_{1}^{*} \phi_{0}^{\prime}(x) \sin \phi_{0}+\mathcal{O}\left(\varepsilon^{2}\right) .
$$

Thus the largest eigenvalue for $\mathcal{L}_{\text {pin }}(x ; h, 0,1+\varepsilon)$ is $\Lambda=0+\varepsilon \Lambda_{1}+\mathcal{O}\left(\varepsilon^{2}\right)$ and the eigenfunction is $\psi=\phi_{0}^{\prime}+\varepsilon \psi_{1}+\mathcal{O}\left(\varepsilon^{2}\right)$. The equation for $\Lambda_{1}$ and $\psi_{1}$ is

$$
\mathcal{L}_{0} \psi_{1}=\Lambda_{1} \phi_{0}^{\prime}+f_{0}(x), \quad \text { where } \quad f_{0}(x)=\left\{\begin{aligned}
-x_{1}^{*} \sin \phi_{0}\left(\phi_{0}^{\prime}\right)^{2}, & x<-L \\
\left(\cos \phi_{0}-\phi_{11} \sin \phi_{0}\right) \phi_{0}^{\prime}, & |x|<L \\
x_{1}^{*} \sin \phi_{0}\left(\psi_{0}^{\prime}\right)^{2}, & x>L
\end{aligned}\right.
$$

From $(24)$ and the fact that $\mathcal{L}_{0} \phi_{0}^{\prime}=0$, it follows that

$$
\begin{array}{lll}
\mathcal{L}_{0} \phi_{11}=\sin \phi_{0}, & \text { hence } & \mathcal{L}_{0} \phi_{11}^{\prime}=2\left(\cos \phi_{0}-\phi_{11} \sin \phi_{0}\right) \phi_{0}^{\prime} \\
\mathcal{L}_{0} \phi_{0}^{\prime}=0, & \text { hence } & \mathcal{L}_{0} \phi_{0}^{\prime \prime}=-\sin \phi_{0}\left(\phi_{0}^{\prime}\right)^{2} .
\end{array}
$$

Thus,

$$
f_{0}(x)=\mathcal{L}_{0}\left\{\begin{aligned}
x_{1}^{*} \phi_{0}^{\prime \prime}(x), & x<-L, \\
\frac{1}{2} \phi_{11}^{\prime}(x), & |x|<L \\
-x_{1}^{*} \phi_{0}^{\prime \prime}(x), & x>L .
\end{aligned}\right.
$$

To find $\Lambda_{1}$, we multiply the eigenvalue equation (25) with $\phi_{0}^{\prime}$, integrate it, use integration by parts and $\mathcal{L}_{0} \phi_{0}^{\prime}=0$ and get

$$
\Lambda_{1} \int_{-\infty}^{\infty}\left(\phi_{0}^{\prime}\right)^{2} d x=2 x_{1}^{*}\left[\left(\phi_{0}^{\prime \prime}(L)\right)^{2}-\phi_{0}^{\prime \prime \prime}(L) \phi_{0}^{\prime}(L)\right]-\phi_{11}^{\prime \prime}(L) \phi_{0}^{\prime}(L)+\phi_{11}^{\prime}(L) \phi_{0}^{\prime \prime}(L) .
$$

with the explicit expressions for $\phi_{0}$ and $\phi_{1}$, we get the expression in the Lemma.

As the linearisation $\mathcal{L}_{0}$ about the sine-Gordon fluxon has exactly one eigenvalue (the one at zero), it follows immediately that if the perturbed linear operator has more eigenvalues, they have come out of the continuous spectrum, hence they are near -1 . 


\section{References}

[1] M.J. Ablowitz, D.J. Kaup, A.C. Newell, and H. Segur, Method for Solving the Sine-Gordon Equation, Phys. Rev. Lett. 30 (1973), p. 1262.

[2] H. Akoh, S. Sakai, A. Yagi and H. Hayakawa, Real time fluxon dynamics in josephson transmission line, IEEE Trans. Magn. 21 (1985), pp. 737-740.

[3] O.Yu. Andreeva, T.L. Boyadjiev, and Yu.M. Shukrinov, Vortex structure in long Josephson junction with two inhomogeneities, Physica C 460-462 (2007), pp. 1315-1316.

[4] A. Benabdallah, J.G. Caputo, and N. Flytzanis, The window Josephson junction: a coupled linear nonlinear system, Physica D 161 (2002), pp. 79-101.

[5] A. Benabdallah and J.G. Caputo, Influence of the passive region on Zero Field Steps for window Josephson junctions, J. Appl. Phys. 92 (2002), pp. 3853-3862.

[6] T.L. Boyadjiev, E.G. Semerdjieva, and Yu.M. Shukrinov, Common features of vortex structure in long exponentially shaped Josephson junctions and Josephson junctions with inhomogeneities, Physica C 460-462 (2007), pp. 1317-1318.

[7] T.L. Boyadjiev, O.Yu. Andreeva, E.G. Semerdjieva, and Yu.M. Shukrinov, Created by current states in long Josephson junctions, Europhys. Lett. 83, (2008), p. 47008.

[8] J.G. Caputo, N. Efraimidis, N. Flytzanis, N. Lazaridis, Y. Gaididei, I. Moulitsa, and E. Vavalis, Static properties and waveguide modes of a wide lateral window Josephson Junction, Int. J. Mod. Phys. C 11 (2000), pp. 493-518.

[9] L.D. Carr, K.W. Mahmud, and W.P. Reinhardt, Tunable tunneling: An application of stationary states of Bose-Einstein condensates in traps of finite depth, Phys. Rev. A 64 (2001), p. 033603.

[10] G. Derks, A. Doelman, S.A. van Gils, and H. Susanto, Stability analysis of $\pi$-kinks in a 0- $\pi$ Josephson junction, SIAM J. Appl. Dyn. Systems 6 (2007), pp. 99-141.

[11] G. Derks, A. Doelman, S.A. van Gils, and T. Visser, Travelling waves in a singularly perturbed sine-Gordon equation, Physica D 180 (2003), pp. 40-70.

[12] Roy H. Goodman and Richard Haberman, Chaotic Scattering and the n-Bounce Resonance in Solitary-Wave Interactions, Phys. Rev. Lett. 98 (2007), p. 104103.

[13] Roy H. Goodman and Michael I. Weinstein, Stability and instability of nonlinear defect states in the coupled mode equations - analytical and numerical study, Phys. D. 237 (2008), pp. 2731-2760.

[14] P.J.A. van Heijster, A. Doelman, T.J. Kaper, Y. Nishiura, K.-I. Ueda, Pinned fronts in heterogeneous media of jump type, Preprint, see http://www.dam.brown.edu/people/heijster/ (2010).

[15] Yu.S. Kivshar, A.M. Kosevich, and O.A. Chubykalo, Finite-size effects in fluxon scattering by an inhomogeneity, Phys. Lett. A 129 (1988), pp. 449-452.

[16] Yuri S. Kivshar and Boris A. Malomed, Dynamics of solitons in nearly integrable systems, Rev. Mod. Phys. 61 (1989), pp. 763-915; ibid., 63 (1991), p. 211 (Addendum).

[17] Yuri S. Kivshar, Zhang Fei, and Luis Vázquez, Resonant soliton-impurity interactions, Phys. Rev. Lett. 67 (1991), p. 1177. 
[18] C.J.K. Knight, Microresistor pinning of $2 k \pi$-fluxons in long Josephson junctions., MMath thesis, University of Surrey (2008).

[19] C.J.K. Knight, G. Derks, A. Doelman, and H. Susanto, Stability of stationary fronts in inhomogeneous wave equations, in preparation.

[20] E. Mann, Systematic perturbation theory for sine-Gordon solitons without use of inverse scattering methods, J. Phys. A: Math. Gen. 30 (1997), pp. 1227-1241.

[21] R. Marangell, C.K.R.T. Jones, and H. Susanto, Localized standing waves in inhomogeneous Schrodinger equations, Nonlinearity 23 (2010), pp. 2059.

[22] D.W. McLaughlin and A.C. Scott, Perturbation analysis of fluxon dynamics, Phys. Rev. A 18 (1978), pp. 1652-1679.

[23] N.G. Parker, Numerical Studies of Vortices and Dark Solitons in Atomic Bose-Einstein Condensates, PhD thesis, Durham University (2004)

[24] B. Piette, W.J. Zakrzewski, and J. Brand, Scattering of topological solitons on holes and barriers, J. Phys. A 38 (2005), pp. 10403-10412.

[25] B. Piette and W.J. Zakrzewski, Dynamical properties of a Soliton in a Potential Well, J. Phys. A 40 (2007), pp. 329-346.

[26] B. Piette and W.J. Zakrzewski, Scattering of Sine-Gordon kinks on potential wells, J. Phys. A 40 (2007), pp. 5995-6010.

[27] H. Sakaguchi and M. Tamura, Scattering and Trapping of Nonlinear Schrödinger Solitons in External Potentials, J. Phys. Soc. Japan 73 (2004), pp. 503-506.

[28] S. Sakai, H. Akoh and H. Hayakawa, Fluxon transfer devices, Japan. J. Appl. Phys. 24 (1985), p. L771.

[29] I.L. Serpuchenko and A.V. Ustinov, Experimental observation of the fine structure on the currentvoltage characteristics of long Josephson junctions with a lattice of inhomogeneities, Sov. Phys. JETP Lett. 46 (1987), p. 549 [Pisma Zh. Eksp. Teor. Fiz. 46 (1987) 435].

[30] H. Susanto, S. A. van Gils, T. P. P. Visser, Ariando, H. J. H. Smilde, and H. Hilgenkamp, Static semifluxons in a long Josephson junction with $\pi$-discontinuity points, Phys. Rev. B. 68 (2003), pp. 104501-104508.

[31] E.C. Titchmarsh, Eigenfunction expansions associated with second-order differential equations, 2nd edition, Oxford University Press, 1962.

[32] A.N. Vystavkin, Yu.F. Drachevskii, V.P. Koshelets, and I.L. Serpuchenko, First observation of static bound states of fluxons in long Josephson junctions with inhomogeneities, Sov. J. Low Temp. Phys. 14 (1988), pp. 357-358 [Fiz. Nizk. Temp. 14 (1988), pp. 646-649.]

[33] M. Weides, M. Kemmler, E. Goldobin, H. Kohlstedt, R. Waser, D. Koelle, and R. Kleiner, 0- $\pi$ Josephson tunnel junctions with ferromagnetic barrier, Phys. Rev. Lett. 97 (2006), p. 247001.

[34] M. Weides, H. Kohlstedt, R. Waser, M. Kemmler, J. Pfeiffer, D. Koelle, R. Kleiner, and E. Goldobin, Ferromagnetic $0-\pi$ Josephson junctions, App. Phys. A 89, (2007) pp. 613-617. 